\title{
Nonrelativistic bound states at finite temperature. II. Muonic hydrogen
}

\author{
Miguel Angel Escobedo and Joan Soto \\ Departament d'Estructura i Constituents de la Matèria and Institut de Ciències del Cosmos, Universitat de Barcelona, \\ Diagonal 647, E-08028 Barcelona, Catalonia, Spain \\ (Received 6 August 2010; published 14 October 2010)
}

\begin{abstract}
We illustrate how to apply modern effective field-theory techniques and dimensional regularization to factorize the various scales, which appear in QED bound states at finite temperature. We focus here on the muonic hydrogen atom. Vacuum polarization effects make the physics of this atom at finite temperature very close to that of heavy quarkonium states. We comment on the implications of our results for these states in the quark gluon plasma. In particular, we estimate the effects of a finite-charm quark mass in the dissociation temperature of bottomonium.
\end{abstract}

DOI: 10.1103/PhysRevA.82.042506

\section{INTRODUCTION}

In a previous paper [1], we showed how to apply modern effective field-theory (EFT) techniques to the hydrogen atom at finite temperature. They provide a systematic way to separate the physics occurring at the various dynamical scales involved in that system, which makes calculations simple and transparent. The main motivation of that work was to pave the way for a QCD-based quantitative study of heavy quarkonium states in the quark gluon plasma (several works in this direction have recently appeared in the literature [2-7]), which share a number of important features with the hydrogen atom. The main qualitative difference, as far as the bound-state dynamics is concerned, between heavy quarkonium states and the hydrogen atom is that vacuum polarization effects in the latter are very suppressed. For muonic hydrogen, however, the vacuum polarization effects provide the leading corrections to the Coulomb potential, as is the case for heavy quarkonium states. This is our main motivation to study muonic hydrogen in detail here.

Muonic hydrogen is under current research at the Paul Scherrer Institute in the so-called muonic hydrogen Lamb shift experiment [8]. It allows for precision tests of QED, which, among other things, probe the electromagnetic structure of the proton [9] or the size of the proton [10]. Theoretical calculations, on one hand, have achieved an impressive precision [11-16], and a number of experimental results are available $[17,18]$. However, thermal effects on this atom due to blackbody radiation, or to electron-positron plasmas, have not been considered to our knowledge, either theoretically or experimentally. Current experimental facilities may now produce electron-positron plasmas [19], which also are the target of intense theoretical studies [20,21] (see Ref. [22] for a recent review). Making muonic hydrogen atoms slowly travel through an electron-positron plasma would be an ideal experiment to probe how well we understand thermal effects in nonrelativistic bound states at relatively high temperatures. Recall that an analogous experiment at lower temperature with blackbody radiation on Rydberg atoms [23] first detected thermal level shifts in the early 1980s. This would mean a further step in taking advantage of the similarities of the electron-positron plasma with the quark-gluon plasma (see Refs. [24,25] for reviews) in order to learn about the nontrivial properties of the latter, as has already been advocated by some authors [26].

In the center-of-mass frame, the proton of a muonic hydrogen is essentially at rest, and the muon moves at small velocities $v \lesssim \alpha \ll 1$. Hence, the relevant scales at zero
PACS number(s): 31.30.jf, 11.10.St, 11.10.Wx, 12.20.Ds

temperature are those of a nonrelativistic system [27]: the muon mass $m_{\mu}$ (hard), the typical momentum $p$, which is of the order $m_{\mu} \alpha / n$ (soft), and the energy $E \sim m_{\mu} \alpha^{2} / n^{2}$ (ultrasoft), $n$ being the principal quantum number. Unlike the hydrogen atom case, vacuum polarization effects introduce a new scale in the muonic hydrogen atom, the electron mass $m_{e}$, which is of the order of the soft scale for the lower-lying states $(n=1,2)$, but larger for the remaining ones. At finite temperature, further scales are introduced, not only $T$, the temperature, but also $e T \sim m_{D}$, the Debye mass, and others that will be discussed later. In order to efficiently deal with the physics at each of these scales, we will use the effective theories of nonrelativistic QED (NRQED) [27], suitable for energies much smaller than the hard scale, potential NRQED (pNRQED) [28], suitable for energies much smaller than the soft scale, and hard thermal loop (HTL) effective theory [29], suitable for energies much smaller than the temperature, in a way analogous to Ref. [1]. Recall that pNRQED enormously facilitates the iteration of the Coulomb potential in ultrasoft contribution (at scale $E$ ), and the HTL action does the same for soft thermal photon resummations at scale $e T$. When scales $e T$ and $E$ coincide, the combination of pNRQED and HTL is crucial in order to consistently obtain both the iteration of the Coulomb potential and the resummation of soft thermal photons.

We will use the real-time formalism [30], which is mandatory for the study of the propagation of a (nonthermalized) nonrelativistic system in a thermal bath. We will restrict ourselves to temperatures much smaller than the muon mass, and hence, the thermal bath does not affect the free muon propagator, which remains the same as at zero temperature. The same holds true for the free proton propagator, which will be further approximated by that of a static source. Thermal propagators will, in general, be necessary for the photons, electrons, and positrons. Recall that, in the real-time formalism, a doubling of degrees of freedom is required to properly account for the thermal propagation. External propagators can only correspond to type-1 fields, vertices contain either type-1 fields or type- 2 fields. Propagators can be $11,12,21$, or 22 . When drawing Feynman diagrams, we will understand that all possible types of vertices and propagators compatible with a given diagram are added up, and will not display each type explicitly (for muons and protons only the 11 propagator must be considered). The techniques and results we will use have been reviewed in Ref. [31]. We reproduced the basic ones in Appendix B. 
We distribute the paper as follows. In Sec. II, we study the ideal case in which the electron mass is set to zero $\left(m_{e}=0\right)$. This not only makes calculations simpler, but also makes the system closer to the heavy quarkonium case. In Sec. III, we focus on the actual case $m_{e} \neq 0$. These two sections are divided into subsections in which the cases $T \ll p, T \sim p$, and $T \gg p$ are studied, $p$ being the typical relative momentum in the bound state (or the inverse Bohr radius). Section IV is devoted to discussion and conclusions.

\section{II. $m_{e}=0 \mathrm{CASE}$}

Let us first consider an ideal case in which the electron mass $m_{e}$ is taken to be zero. This case is in fact closer to the one in heavy quarkonium states, particularly in charmonium, than the actual case with $m_{e} \neq 0$, which we will study in Sec. III. It has already been discussed in the past in order to clarify subtle issues on the renormalization group structure of nonrelativistic effective theories [32].

\section{A. $p \gg T$}

For temperatures much smaller than the soft scale $\left(m_{\mu} \alpha\right.$ in this case), we can study the thermal effects in the atom starting from the $\mathrm{pNRQED} \mathrm{Lagrangian}$ at zero temperature, up to exponentially suppressed contributions $\sim e^{-p / T}$. This means that the potentials are the same as the ones at zero temperature. The only difference, with respect to the hydrogen atom case, is that these potentials now contain $O(\alpha)$ corrections due to vacuum polarization effects produced by virtual electron-positron pairs. As in the hydrogen atom, the ultrasoft photons, electrons, and positrons are responsible for the finite-temperature effects. Then, our starting point in this section is Eq. (6) from Ref. [33],

$$
\begin{aligned}
L_{\mathrm{pNRQED}}= & -\int d^{3} \mathbf{x} \frac{1}{4} F_{\mu v}(t, \mathbf{x}) F^{\mu v}(t, \mathbf{x}) \\
& +\int d^{3} \mathbf{r} d^{3} \mathbf{R} S^{\dagger}(t, \mathbf{r}, \mathbf{R})\left[i \partial_{0}+\frac{\nabla^{2}}{2 m_{\mu}}+\frac{\alpha}{|\mathbf{r}|}\right. \\
& +\frac{\nabla^{4}}{8 m_{\mu}^{3}}+\frac{e^{2}}{m_{\mu}^{2}}\left(-\frac{c_{D}}{8}+4 d_{2}\right) \delta^{3}(\mathbf{r}) \\
& \left.+i c_{S} \frac{\alpha}{4 m_{\mu}^{2}} \sigma \cdot\left(\frac{\mathbf{r}}{|\mathbf{r}|^{3}} \times \nabla\right)\right] S(t, \mathbf{r}, \mathbf{R}) \\
& +\int d^{3} \mathbf{r} d^{3} \mathbf{R} S^{\dagger}(t, \mathbf{r}, \mathbf{R}) e \mathbf{r} \cdot \mathbf{E}(t, \mathbf{R}) S(t, \mathbf{r}, \mathbf{R}) \\
& +\int d^{3} \mathbf{x} \bar{e}(t, \mathbf{x}) i \gamma^{\mu} D_{\mu} e(t, \mathbf{x}),
\end{aligned}
$$

where $S(t, \mathbf{r}, \mathbf{R})$ is the muon wave-function field, $\mathbf{r}$ being its distance to the proton and $\mathbf{R}$ being the position of the proton; $e(t, \mathbf{x})$ is the electron Dirac field. $\alpha=e^{2} / 4 \pi$ is the electromagnetic coupling constant, and $c_{0}, c_{s}$, and $d_{2}$ are matchings coefficients, which can be found at one loop in Ref. [34].

Let us separate the cases $T \lesssim E$ and $T \gg E$, which are analyzed in Secs. II A 1 and II A 2.

$$
\text { 1. } T \lesssim E
$$

In this case, the leading temperature-dependent contributions are given by the diagram in Fig. 1, in an analogous way to the hydrogen atom case. Virtual ultrasoft electron-positron pairs give rise to $O(\alpha)$ corrections, and no soft thermal photon

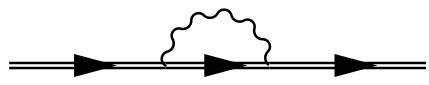

FIG. 1. Muonic hydrogen atom self-energy at leading order (due to ultrasoft photons). The double line stands for the propagator of the atom, and the wavy line stands for the thermal propagator of the transverse photons. The vertex corresponds to the dipole term in the multipole expansion, see Eq. (1).

resummation is necessary at scale $E$. Hence, there is no qualitative difference with respect to the hydrogen atom, and we will not discuss it further. We refer to Ref. [1] for the relevant formulas for the spectrum and decay widths. ${ }^{1}$

\section{2. $T \gg E$}

In this case, scale $T$ can be integrated out before calculating the spectrum and decay widths we call the resulting effective theory $\mathrm{pNRQED}{ }_{>T}$ (for further explanations about the notation, see Appendix A). In the photon and electron-positron sectors, this gives rise to the HTL action [29]. In the atom sector, the pNRQED Lagrangian gets additional temperaturedependent potentials. At leading order (LO) in $\alpha$, they arise from the diagram in Fig. 1 upon expanding $E-H$ in the integrals, and have been calculated in Ref. [1],

$$
\begin{aligned}
\delta V_{T}^{(\mathrm{LO})}= & \frac{\alpha \pi T^{2}}{3 m_{\mu}}-\frac{4 \alpha^{2}}{3 m_{\mu}^{2}} \delta^{3}(\mathbf{r})\left[\frac{1}{\epsilon}+\ln \left(\frac{\mu}{2 \pi T}\right)\right. \\
& \left.+\frac{5}{6}+\ln (2 \pi)\right]+O\left(\frac{\alpha r^{2} E^{4}}{T}\right),
\end{aligned}
$$

$H \sim E \sim \alpha / r$. Possible $O\left(\alpha r^{4} E T^{4}\right)$ terms arising from higher orders in the multipole expansion cancel out. Note that the preceding dominant contribution is a constant mass shift, and the $r$-dependent part is $\left(m_{\mu} \alpha^{2} / n^{2} T\right)^{2}$ suppressed. Hence, vacuum polarization corrections to the photon propagator may compete with the $r$-dependent part of the LO potential displayed previously and must be calculated. From the diagrams in Fig. 2, we obtain the next-to-leading order (NLO) in $\alpha$,

$$
\begin{aligned}
\delta V_{T}^{(\mathrm{NLO})}= & -\frac{3 \alpha}{2 \pi} \zeta(3) T m_{D}^{2} r^{2}+\frac{i \alpha T m_{D}^{2}}{6} r^{2}\left[\frac{1}{\epsilon}+\gamma+\ln \pi\right. \\
& \left.-\ln \frac{T^{2}}{\mu^{2}}+\frac{2}{3}-4 \ln 2-2 \frac{\zeta^{\prime}(2)}{\zeta(2)}\right]
\end{aligned}
$$

${ }^{1}$ In the case $E \gg T$, the formulas presented in Ref. [1] provide the leading contribution for $T \gg \alpha E$ only. For $T \lesssim \alpha E$, additional contributions exist [35].

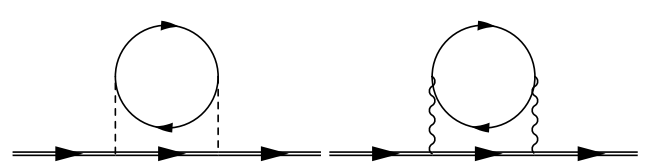

FIG. 2. Muonic hydrogen atom self-energy at NLO (due to ultrasoft photons, electrons, and positrons). The solid line stands for the thermal Dirac propagator of electrons and positrons, and the dashed line stands for the propagator of the longitudinal $\left(A_{0}\right)$ photons. The remaining lines are as in Fig. 1. 


$$
\begin{aligned}
& -\frac{i \alpha m_{D}^{2}}{32 m_{\mu}}\left[\frac{1}{\epsilon}+c+2 \gamma+2 \ln \left(\frac{\mu}{T}\right)\right] \\
& +O\left(\frac{\alpha r^{2} m_{D}^{2} E^{2}}{T}, \frac{\alpha r^{2} m_{D}^{4}}{T}\right),
\end{aligned}
$$

with $m_{D}^{2}=(e T)^{2} / 3$ and $c$ a numerical constant. The computations have been done in dimensional regularization (DR) with $\epsilon=(4-d) / 2 \rightarrow 0$. The first and second lines of this result also appears in an analogous calculation that has already been carried out in the static limit of the QCD case [6]. The third line is subleading. We have displayed it to match the precision of Eq. (2) when $e T \sim E$. In order to eventually check the correct cancellation of the $1 / \epsilon$ poles, we have calculated analytically the leading infrared (IR) behavior in Appendix C. The constant $c$ remains unknown. We see that indeed $\delta V_{T}^{(\mathrm{NLO})}$ competes in size with $\delta V_{T}^{(\mathrm{LO})}$, except for the global energy shift given by the first term in Eq. (2). In fact, it provides the dominant term in the potential for the energy of the photon transitions between two states belonging to this case. The IR divergences arising earlier are canceled by ultraviolet (UV) divergences arising from contributions at smaller scales $(E, e T, \ldots)$. These contributions are hard to calculate in the general case because HTL propagators must be used for the ultrasoft photons and the Coulomb potential must be kept unexpanded in the atom propagator. At these scales, however, the Bose distribution can be expanded, which simplifies somewhat the calculations, and produces the so-called Bose enhancement, see Eqs. (B8) and (B10). The dominant contribution arises from the diagrams of Fig. 3. We have only been able to work out an analytic expression for cases $e T \ll E$ and $e T \gg E$, which will be discussed as follows and for its UV behavior. Technical details for the latter are shown in Appendix C.

Before discussing the two cases, which allow to proceed further analytically, we display the energy shift and decay width induced by the temperature-dependent potentials (2) and (3) at LO in perturbation theory,

$$
\begin{aligned}
& \delta E_{n}^{T}=\frac{\alpha \pi T^{2}}{3 m_{\mu}}-\frac{2 \alpha}{3 \pi m_{\mu}^{2}}\left|\phi_{n}(\mathbf{0})\right|^{2}\left[\frac{1}{\epsilon}+\ln \left(\frac{\mu}{2 \pi T}\right)\right. \\
& \left.+\frac{5}{6}+\ln (2 \pi)\right]-\frac{3 \alpha}{2 \pi} \zeta(3) T m_{D}^{2}\left\langle r^{2}\right\rangle_{n} \\
& +O\left(\frac{\alpha r^{2} E^{4}}{T}, \frac{\alpha r^{2} m_{D}^{4}}{T}\right), \\
& \delta \Gamma_{n}^{T}=-\frac{\alpha T m_{D}^{2}\left\langle r^{2}\right\rangle_{n}}{3}\left[\frac{1}{\epsilon}+\gamma+\ln \pi-\ln \frac{T^{2}}{\mu^{2}}+\frac{2}{3}\right. \\
& \left.-4 \ln 2-2 \frac{\zeta^{\prime}(2)}{\zeta(2)}\right]+\frac{\alpha m_{D}^{2}}{16 m_{\mu}}\left[\frac{1}{\epsilon}+c+2 \gamma\right. \\
& \left.+2 \ln \left(\frac{\mu}{T}\right)\right]+O\left(\frac{\alpha r^{2} E^{4}}{T}, \frac{\alpha r^{2} m_{D}^{4}}{T}\right),
\end{aligned}
$$

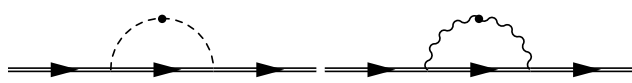

FIG. 3. Further contributions to the muonic hydrogen atom selfenergy when $p \gg T \gg E$. The wavy line and the dashed line with a blob are the HTL propagators for the transverse and longitudinal photons, respectively. in which $\left\langle r^{2}\right\rangle_{n}=\frac{n^{2}}{2 m_{\mu}^{2} \alpha^{2}}\left[5 n^{2}+1-3 l(l+1)\right], n$ and $l$ being the principal and angular momentum quantum numbers here. The labels $n, m, \ldots$ are also used through the paper as a short-hand notation for the whole ensemble of quantum numbers of a given Coulomb state, either bound or in the continuum, and $\phi_{n}(\mathbf{0})$ is the wave function at the origin. The previous contributions are to be added to the ones coming from lower scales, which we discuss as follows in two particular cases that share the feature that the lower-energy scales are hierarchically ordered, and hence the method of the integration by regions can be used $[36,37]$

(1) $E \gg e T$. In this case, the loop integral is dominated by energy and momentum $\sim E$ for which $e T$ can be treated as a perturbation. At LO in the HTL expansion, we obtain

$$
\begin{aligned}
\delta E_{n}^{E}= & \frac{2 \alpha}{3 \pi} \sum_{m}|\langle n|\mathbf{v}| m\rangle|^{2}\left(E_{n}-E_{m}\right) \\
\times & {\left[\frac{1}{\epsilon}+\ln \left(\frac{\mu}{\left|E_{n}-E_{m}\right|}\right)+\frac{5}{6}-\gamma+\ln (2 \pi)\right] } \\
- & \frac{\alpha \pi T m_{D}^{2}}{3}\left\langle r^{2}\right\rangle_{n}+O\left(\frac{\alpha r^{2} T m_{D}^{4}}{E^{2}}\right), \\
\delta \Gamma_{n}^{E}= & \frac{4 \alpha^{3} T}{3 n^{2}}+\frac{\alpha T m_{D}^{2}}{3} \sum_{m}|\langle n|\mathbf{r}| m\rangle|^{2} \\
& \times\left[\frac{1}{\epsilon}-2 \ln \frac{\left|E_{n}-E_{m}\right|}{\mu}+\frac{11}{3}-\ln 4\right. \\
& -\gamma+\ln (\pi)]+O\left(\frac{\alpha r^{2} T m_{D}^{4}}{E^{2}}\right) .
\end{aligned}
$$

We observe that the LO IR divergences appearing at scale $T$ in Eqs. (4) and (5) are canceled by the UV divergences in Eqs. (6) and (7), respectively. Note that the subleading IR divergence at scale $T$, in the second line of Eq. (5), is very much suppressed in this case $\left(m_{\mu} \alpha^{5} \gg \alpha m_{D}^{2} / m_{\mu}\right)$. A similar calculation for the heavy quarkonium case has been presented in Ref. [38]. Technical details can be found in Appendix Sec. C 2. Here, we only mention that a collinear region exists that contributes at this order.

Upon summing up the contributions from both energy regions, namely, Eqs. (4) and (6) for the energy and Eqs. (5) and (7) for the decay width, finite results are obtained at the desired order,

$$
\begin{aligned}
\delta E_{n}= & \frac{\alpha \pi T^{2}}{3 m_{\mu}}+\frac{2 \alpha}{3 \pi}\left|\phi_{n}(0)\right|^{2}\left[\ln \left(\frac{2 \pi T}{\left|E_{n}\right|}\right)-\gamma\right] \\
& +\frac{2 \alpha}{2 \pi} \sum_{m}|\langle n|\mathbf{v}| m\rangle|^{2}\left(E_{n}-E_{m}\right) \ln \frac{\left|E_{n}\right|}{\left|E_{n}-E_{m}\right|} \\
& -\frac{\alpha T m_{D}^{2}\left\langle r^{2}\right\rangle_{n}}{\pi}\left(\frac{3 \zeta(3)}{2}+\frac{\pi^{2}}{3}\right) \\
& +O\left(\frac{\alpha r^{2} E^{4}}{T}, \frac{\alpha r^{2} T m_{D}^{4}}{E^{2}}\right),
\end{aligned}
$$




$$
\begin{aligned}
\delta \Gamma_{n}= & \frac{4 \alpha^{3} T}{3 n^{2}}+\frac{2 \alpha T m_{D}^{2}\left\langle r^{2}\right\rangle_{n}}{3}\left[\ln \frac{T}{\left|E_{n}\right|}-\gamma+\frac{3}{2}\right. \\
& \left.+\ln 2+\frac{\zeta^{\prime}(2)}{\zeta(2)}\right]-\frac{2 \alpha T m_{D}^{2}}{3} \sum_{m}|\langle n|\mathbf{r}| m\rangle|^{2} \\
& \times \ln \frac{\left|E_{n}-E_{m}\right|}{\left|E_{n}\right|}+O\left(\frac{\alpha r^{2} E^{4}}{T}, \frac{\alpha r^{2} T m_{D}^{4}}{E^{2}}\right) .
\end{aligned}
$$

(2) $e T \gg E$. In this case, the loop integral is dominated by energy and momentum $\sim e T$ for which $E$ can be treated as a perturbation. At LO in the energy expansion, we obtain a contribution, which is equivalent to adding a new term to the potential that goes like $m_{D}^{2} r^{2}$,

$$
\begin{gathered}
\delta E_{n}^{e T^{\mathrm{LO}}}=\frac{\alpha m_{D}^{3}}{6}\left\langle r^{2}\right\rangle_{n}+O\left(\alpha^{2} r^{2} m_{D}^{2} T\right) \\
\delta \Gamma_{n}^{e T^{\mathrm{LO}}}=\frac{\alpha T m_{D}^{2}}{3}\left\langle r^{2}\right\rangle_{n}\left(\frac{1}{\epsilon}-\gamma+\ln \pi+\ln \frac{\mu^{2}}{m_{D}^{2}}+\frac{5}{3}\right) \\
+O\left(\alpha^{2} r^{2} m_{D}^{2} T\right) .
\end{gathered}
$$

We observe that the LO IR divergence appearing at scale $T$ is canceled by the earlier UV divergence. An analogous contribution has also been calculated in the static limit of QCD [6].

At NLO in the energy expansion, we have restricted ourselves to compute the UV divergence analytically,

$$
\begin{aligned}
\delta \Gamma_{n}^{e T^{\mathrm{NLO}}}= & -\frac{\alpha m_{D}^{2}}{16 m_{\mu}}\left[\frac{1}{\epsilon}+c^{*}-2 \ln \left(\frac{m_{D}}{\mu}\right)\right] \\
& +O\left(\alpha r^{2} E^{2} T\right) .
\end{aligned}
$$

It cancels the IR divergence in the second line of Eq. (3), as it should ( $c^{*}$ is an unknown constant that can be of order $1 / \alpha^{1 / 2}$ because of Bose enhancement).

Fortunately, the contribution of the loop integral for energy and momenta $\sim E$ is subleading. The calculation at that scale may even require nonperturbative techniques if $E$ gets close to scale $e^{2} T[30,39,40]$.

Summing up the contributions from the $T$ energy region and from the $m_{D}$ energy region, namely, Eqs. (4) and (10) for the energy and Eqs. (5) and (11) for the decay width, the leading thermal effects for this situation are obtained,

$$
\delta E_{n}=\frac{\alpha \pi T^{2}}{3 m_{\mu}}+\frac{\alpha m_{D}^{3}\left\langle r^{2}\right\rangle_{n}}{6}+O\left(\alpha r^{2} E^{2} T\right),
$$

$$
\begin{aligned}
\delta \Gamma_{n}= & \frac{2 \alpha T m_{D}^{2}\left\langle r^{2}\right\rangle_{n}}{3}\left[\ln \frac{T}{m_{D}}-\gamma+\frac{1}{2}+2 \ln 2+\frac{\zeta^{\prime}(2)}{\zeta(2)}\right] \\
& -\frac{2 \alpha m_{D}^{2}}{16 m_{\mu}}\left(\ln \frac{T}{m_{D}}+\frac{c^{*}-c}{2}-\gamma\right)+O\left(\alpha r^{2} E^{2} T\right) .
\end{aligned}
$$

\section{B. $T \sim p$}

Since $m_{\mu} \gg T$ still holds, our starting point is the NRQED Lagrangian at $T=0$ [27] (this is correct up to exponentially small contributions $\sim e^{-m_{\mu} / T}$ ),

$$
\begin{aligned}
\mathcal{L}= & \psi^{+}\left[i D^{0}+\frac{\mathbf{D}^{2}}{2 m_{\mu}}+\frac{\mathbf{D}^{4}}{8 m_{\mu}^{3}}+c_{F} e \frac{\boldsymbol{\sigma} \cdot \mathbf{B}}{2 m_{\mu}}+c_{D} e \frac{|\nabla \cdot \mathbf{E}|}{8 m_{\mu}^{2}}\right. \\
& \left.+i c_{S} e \frac{\boldsymbol{\sigma}(\mathbf{D} \times \mathbf{E}-\mathbf{E} \times \mathbf{D})}{8 m_{\mu}^{2}}\right] \psi+N^{+} i D^{0} N-\frac{1}{4} F_{\mu \nu} F^{\mu \nu} \\
& +\frac{d_{2}}{m_{\mu}^{2}} F_{\mu \nu} D^{2} F^{\mu \nu}+\bar{e} i \gamma^{\mu} D_{\mu} e .
\end{aligned}
$$

where $\psi$ is the muon Pauli-spinor field.

Since $T \sim p \gg E$, we can integrate out scales $T$ and $p$, which leads to what we call $\mathrm{pNRQED}_{T}$, the suitable effective theory for scale $E$, which is similar to the one that has already been introduced in Sec. II A, with the only difference that now $T$ is of the same order as the cutoff of the effective theory. In the photon and electron-positron sectors, we have the standard HTL. In the atom sector, temperature-dependent potentials are induced. Recall that, at scale $T$, there is no enhancement, and vacuum polarization effects due to electron-positron pairs are always suppressed by $\alpha$. Hence, the leading potential will still be the Coulomb potential, but the first $\alpha$ correction to it will already be temperature dependent. This is given by the diagram in Fig. 4.

The temperature-dependent part of the potential induced by the diagram in Fig. 4 is both UV and IR safe in momentum space. However, when it is Fourier transformed to coordinate space, an IR divergence is encountered. The calculation is rather involved, so we only display the final result here, which can be given in terms of one-parameter integrals of special functions. Details are given in Appendix D (the formulas in that appendix have to be used setting $m_{e}=0$ for this case). We obtain,

$$
\begin{aligned}
\delta V_{r}= & -\frac{\alpha m_{D}^{2} r}{4}-\frac{3 \alpha}{2 \pi} \zeta(3) T m_{D}^{2} r^{2}+\frac{\alpha m_{D}^{2}}{4 \pi^{2} T^{2} r} \int_{0}^{\infty} \frac{d u}{u\left(e^{u}+1\right)}\left[-4-4 \rho^{2} u^{2}+\left(\rho^{2} u^{2}+4\right) \cos (\rho u)+\rho u \sin (\rho u)\right. \\
& \left.+\left(6 \rho u+\rho^{3} u^{3}\right) \operatorname{Si}(\rho u)\right]+\frac{i \alpha m_{D}^{2} T r^{2}}{6}\left[\frac{1}{\epsilon}+\gamma+\ln \pi+2 \ln (r \mu)-1\right]-\frac{i 3 \alpha m_{D}^{2}}{2 \pi^{2} T}\left[\frac{1}{2}-\ln (r T)-\ln \pi\right] \\
& +\frac{i 3 \alpha m_{D}^{2}}{\pi^{2} T^{2} r} \int_{0}^{\infty} \frac{d u}{u^{4}} \sin (\rho u)\left[L i_{2}\left(-e^{u}\right)+u \ln \left(1+e^{u}\right)+\frac{\pi^{2}}{12}-\frac{u^{2}}{4}\right]+O\left(\alpha^{3} T\right),
\end{aligned}
$$

where $\rho=2 r T$ and Si stands for sine integral,

$$
\operatorname{Si}(z)=\int_{0}^{z} \frac{\sin t}{t} d t \text {. }
$$

The LO energy correction is obtained by computing the expectation value of the potential of Eq. (16) for the desired state and adding the ultrasoft contribution. 
The calculation in $\mathrm{pNRQED}_{T}$ is identical to one carried out in the second case of Sec. II A (since $E \sim m_{\mu} \alpha^{2}, T \sim p \sim m_{\mu} \alpha$, we have $e T \gg E$ ). Hence, the outcome can be directly read off Eqs. (10) and (11). Notice that the IR divergence in Eq. (16) at first order in quantum-mechanic perturbation theory, induces a contribution that cancels out the UV divergence in Eq. (11), as it should. Also, the contribution from integrating out the scale $m_{D}$ can be encoded in a correction to the potential, summing it up to $\delta V_{r}$, where the following finite result is obtained,

$$
\begin{aligned}
\delta V= & -\frac{\alpha m_{D}^{2} r}{4}-\frac{3 \alpha}{2 \pi} \zeta(3) T m_{D}^{2} r^{2}+\frac{\alpha m_{D}^{3} r^{2}}{6}+\frac{\alpha m_{D}^{2}}{4 \pi^{2} T^{2} r} \int_{0}^{\infty} \frac{d u}{u\left(e^{u}+1\right)}\left[-4-4 \rho^{2} u^{2}+\left(\rho^{2} u^{2}+4\right) \cos (\rho u)+\rho u \sin (\rho u)\right. \\
& \left.+\left(6 \rho u+\rho^{3} u^{3}\right) \operatorname{Si}(\rho u)\right]-\frac{i \alpha m_{D}^{2} T r^{2}}{3}\left[-\ln \left(r m_{D}\right)+\frac{4}{3}-\gamma\right]-\frac{i 3 \alpha m_{D}^{2}}{2 \pi^{2} T}\left[\frac{1}{2}-\ln (r T)-\ln \pi\right] \\
& +\frac{i 3 \alpha m_{D}^{2}}{\pi^{2} T^{2} r} \int_{0}^{\infty} \frac{d u}{u^{4}} \sin (\rho u)\left[L i_{2}\left(-e^{u}\right)+u \ln \left(1+e^{u}\right)+\frac{\pi^{2}}{12}-\frac{u^{2}}{4}\right]+O\left(\alpha^{3} T\right)
\end{aligned}
$$

and hence, at first order in perturbation theory,

$$
\begin{gathered}
\delta E_{n}=\langle n|\operatorname{Re} \delta V| n\rangle, \\
\delta \Gamma_{n}=-2\langle n|\operatorname{Im} \delta V| n\rangle . \\
\text { C. } \boldsymbol{T} \gg \boldsymbol{p}
\end{gathered}
$$

Since $m_{\mu} \gg T$ still holds, we can also start from NRQED at $T=0$. Now, we may proceed by sequentially integrating out first scale $T$ and next scale $p$. After integration of scale $T$, we get an effective theory, which consists of HTL contributions in the photon and electron-positron sectors and of NRQED with temperature-dependent matching coefficients in the atom sector. This NRQED ${ }_{>T}$ in the atom sector is identical to the one that we have in the hydrogen atom case [1], up to order $\alpha$ corrections induced by the electron-positron vacuum polarization.

The next step is to integrate out energy scale $p$, namely, to match NRQED ${ }_{>T}$ to what will be called $\mathrm{pNRQED}_{<T}$, which is expected to produce temperature-dependent potentials. These potentials must be calculated using HTL photon propagators. Let us separate the two following cases:

(1) $e T \sim p$. In this case, the computations can be carried out as in Sec. VB of Ref. [1]. The relevant diagram is similar to Fig. 4, but instead of a photon propagator with a self-energy insertion, we would have to use the tree-level HTL photon propagator (Fig. 5). The only difference, with respect to Ref. [1], is due to the fact that the electron-positron pairs that generated the HTL photon propagators are now

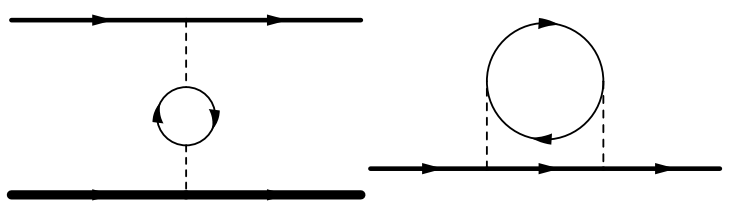

FIG. 4. Leading correction to the Coulomb potential due to vacuum polarization when $T \lesssim p$. The thick and extra-thick solid lines stand for the nonrelativistic propagator of the muon and the static propagator of the proton, respectively. The remaining lines are as in Fig. 2. There is also an LO correction coming from an extra diagram, which is obtained from the second one by changing the muon line by a proton line. taken to be massless, so, in fact, the outcome is simpler: The nontrivial function $g\left(m_{e} \beta\right)$ reduces to $\frac{\pi m_{D}^{2}}{16 \alpha T^{2}}$. Then, we obtain the following LO potential:

$$
V(r, T)=-\frac{\alpha e^{-m_{D} r}}{r}-\alpha m_{D}+i \alpha T \phi\left(m_{D} r\right)+O\left(\frac{\alpha T^{2}}{m_{\mu}}\right),
$$

where

$$
\phi(x)=2 \int_{0}^{\infty} \frac{d z z}{\left(z^{2}+1\right)^{2}}\left[\frac{\sin (z x)}{z x}-1\right] .
$$

This potential coincides with the one first obtained in Ref. [2] for QCD (up to trivial changes in color factors made explicit in Ref. [1]). As in the hydrogen atom case, we can use this result in order to estimate the dissociation temperature, which is $T_{d} \sim m_{\mu} \alpha^{2 / 3} / \ln ^{1 / 3} \alpha$, as anticipated in Ref. [1] for the QCD case.

(2) $e T \gg p$. In this case, $T \gg T_{d}$ always holds. Hence, the imaginary part of the potential is bigger than the real one, so it does not make much sense to speak about bound states anymore.

\section{III. $m_{e} \neq 0$ CASE}

Now, we address the actual case of a nonvanishing electron mass. Although the real muonic hydrogen is not as close to heavy quarkonium systems as the ideal one $\left(m_{e}=0\right)$, it may still be useful to learn about certain aspects of it. In particular, about the role of a finite-charm mass in the bottomonium system, which is analogous to that of a finite-electron mass in muonic hydrogen [41]. This case may then shed light on

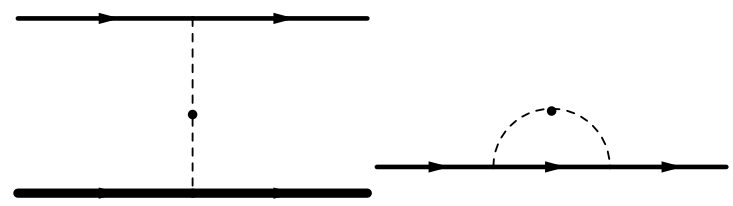

FIG. 5. Leading correction to the Coulomb potential due to vacuum polarization when $T \gg p$. The lines are as in Figs. 3 and 4. 
the effects of the charm quark mass in bottomonium at finite temperature, specially when charm quarks are thermalized. Irrespective of that, muonic hydrogen is a real system that appears in nature, which nowadays is produced in large samples [8], and, therefore, our results may eventually be checked against the experiment.

For actual muonic hydrogen $m_{e} \sim p$ for the lower-lying states $(n=1,2)$, whereas for the remaining states $(n \geqslant 3)$, one may safely consider $m_{e} \gg p$ [41]. Then, let us analyze these two cases separately.

\section{A. Lower-lying states $(n=1,2)$}

As mentioned previously, these two states fulfill $p \sim m_{e}$, and hence relativistic electron-positron pairs must be integrated at the same time as the momentum transfer $p$. In Secs. III B and III C, let us see how this is carried out depending on what the temperature is.

\section{1. $T \ll p$}

As in the massless case, our starting point can be pNRQED. However, now, due to the fact that $m_{e} \sim p$ (rather than $m_{e}=0$ ), the electron-positron pairs have already been integrated out when calculating the potentials, and hence are not active anymore. The situation is then totally analogous to the hydrogen atom, the only difference being that the potentials get $O(\alpha)$ corrections due to virtual electron-positron pairs, the most important of which is the Uehling potential. In other words, the thermal bath contains neither electrons nor positrons, so the thermal effects are only due to the photons, which do not distinguish between electrons and muons. Hence, the results concerning this case can be read off Sec. III of Ref. [1] by making $m \rightarrow m_{\mu}$ [up to $O(\alpha)$ corrections].

\section{2. $T \sim p$}

Again, as in the massless case, we can start with NRQED. Scales $T$ and $m_{e}$ must be integrated out at the same time as energy scale $p$. In the photon sector, which is not sensitive to scale $p$, we get the mass-dependent HTL action (see Sec. V A 1 of Ref. [1]). In the electron and positron sectors, which are not sensitive to scale $p$ either, we get an $\mathrm{NRQED}_{T}$ Lagrangian for each of these particles (see Sec. V A 2 of Ref. [1]). In the atom sector, the potentials now depend on both temperature and electron mass, except for the leading Coulomb potential. The most important correction is a kind of temperature-dependent Uehling potential, which is obtained from the diagram in Fig. 4,

$$
\begin{aligned}
\delta V_{r}= & -\frac{4 \alpha^{2} f\left(m_{e} \beta\right) m_{e}^{2} r}{\pi}-\frac{2 \alpha^{2}}{\pi r} \int_{0}^{\infty} \frac{d u}{\sqrt{u^{2}+1}\left(e^{\beta m_{e} \sqrt{u^{2}+1}}+1\right)}[1-\cos (\sigma u)-\sigma u \operatorname{Si}(\sigma u)] \\
& +\frac{\alpha^{2}}{3 \pi r} \int_{0}^{\infty} \frac{d u \sqrt{u^{2}+1}}{u^{2}\left(e^{\beta m_{e} \sqrt{u^{2}+1}}+1\right)}\left[2-3 \sigma^{2} u^{2}+\left(\sigma^{2} u^{2}-2\right) \cos (\sigma u)+\sigma u \sin (\sigma u)+\sigma^{3} u^{3} \operatorname{Si}(\sigma u)\right] \\
& -\frac{\alpha}{\pi} T m_{D}^{2} r^{2}\left(\beta m_{e}\right)^{3} \int_{0}^{\infty} d u \frac{u \sqrt{u^{2}+1}}{e^{\beta m_{e} \sqrt{u^{2}+1}}+1}+\frac{i 8 \alpha^{2} T^{3} g\left(m_{e} \beta\right) r^{2}}{3 \pi}\left[\frac{1}{\epsilon}+\gamma+\ln \pi+\ln (r \mu)^{2}-1\right] \\
& -\frac{i 4 \alpha^{2} T}{\pi\left(e^{\beta m_{e}}+1\right)}\left[\frac{1}{2}-\ln (r T)-\ln 2-\left(e^{\beta m_{e}}+1\right) \int_{0}^{\infty} \frac{d u}{u\left(e^{\beta m_{e} \sqrt{u^{2}+1}}+1\right)}+\int_{0}^{\infty} \frac{d u e^{-\beta m_{e} u}}{u}\right] \\
& +\frac{i 4 \alpha^{2} T^{3}}{\pi r m_{e}^{3}} \int_{0}^{\infty} \frac{d u}{u^{4}} \sin (\sigma u)\left[L i_{2}\left(-e^{\beta m_{e} \sqrt{u^{2}+1}}\right)+\left(\beta m_{e}\right) \sqrt{u^{2}+1} \ln \left(1+e^{\beta m_{e} \sqrt{u^{2}+1}}\right)+\frac{\pi^{2}}{6}-\frac{\left(\beta m_{e}\right)^{2}}{2}\left(u^{2}+1\right)\right. \\
& \left.-g\left(m_{e} \beta\right)+\frac{\left(\beta m_{e}\right)^{2} u^{2}}{2\left(e^{\beta m_{e}}+1\right)}\right]+\frac{i 4 \alpha^{2} T}{\pi} \int_{0}^{\infty} \frac{d u}{u^{3}}\left[\frac{\operatorname{Sinc}(\sigma u)-1}{e^{\beta m_{e} \sqrt{u^{2}+1}}+1}-\frac{\operatorname{Sinc}(\sigma u)-e^{-8 \beta^{3} m_{e}^{3} u^{3}}}{e^{\beta m_{e}}+1}\right] \\
& +\frac{i 4 \alpha^{2} m_{e}^{2} T r^{2}}{3 \pi\left(e^{\beta m_{e}}+1\right)}\left[\frac{1}{\epsilon}-1+\gamma+2 \ln (r \mu)+\ln \pi\right]-\frac{i 16 \alpha^{2} m_{e}^{2}}{3 \pi T\left(e^{\beta m_{e}}+1\right)} \Gamma(-2 / 3)+O\left(\alpha^{3} T\right),
\end{aligned}
$$

where $\beta=1 / T, \sigma=2 m_{e} r$, and

$$
\begin{gathered}
f\left(m_{e} \beta\right)=\int_{0}^{\infty} d x \frac{x^{2}}{\sqrt{x^{2}+1}\left(e^{\beta m_{e} \sqrt{x^{2}+1}}+1\right)}, \\
g\left(m_{e} \beta\right)=\beta^{2} m_{e}^{2} \int_{0}^{\infty} d x \frac{x}{e^{\beta m_{e} \sqrt{x^{2}+1}}+1} .
\end{gathered}
$$

Further expressions for these functions can be found in Appendix B of Ref. [1]. The computations that lead to Eq. (22) are carried out in Appendix D.

Notice that Eq. (22) has two IR divergences, which, as in the $m_{e}=0$ case, arise when the Fourier transform of the momentum space potential is taken in order to get the coordinate space potential. The IR divergence in the third line of Eq. (22) is similar to the one that appears in Eq. (16) for 
the massless case [with $T^{2} g\left(m_{e} \beta\right)$ instead of $m_{D}^{2}$ ]. The IR divergence in the last line of Eq. (22), however, is proportional to $\frac{m_{e}^{2}}{e^{\beta m_{e}}+1}$, and hence distinct from the $m_{e}=0$ case. It emerges from a region in which not only the three-momentum transfer is small, but also the component of the three-momentum of the electron-positron pair in the loop parallel to the momentum transfer is small. In either case, these IR divergences should cancel against UV divergences in the pNRQED calculation.

The relevant diagram in the pNRQED calculation is again Fig. 3 in which the photon line must be understood as the massdependent HTL propagator (see Appendix E). In the dominant contribution to this diagram, $E-H$ in the atom propagator can be treated as a perturbation (recall that $E-H \sim m_{\mu} \alpha^{2} \ll$ $e T)$. Then, using the fact that $\Delta_{11}\left(k^{0}, \mathbf{k}\right)$ is symmetric with respect to $k^{0} \rightarrow-k^{0}$, we obtain

$$
\delta E_{n}^{e T}=\frac{\alpha m_{D}^{3}}{6}\left\langle r^{2}\right\rangle_{n}+O\left(\alpha r^{2} E m_{D}^{2}\right),
$$

$$
\begin{aligned}
\delta \Gamma_{n}^{e T}= & \left\{\frac{16 \alpha^{2} T^{3}}{3 \pi} g\left(m_{e} \beta\right)\left(\frac{1}{\epsilon}-\gamma+\ln \pi+\ln \frac{\mu^{2}}{m_{D}^{2}}+\frac{5}{3}\right)\right. \\
& +\frac{8 \alpha^{2} m_{e}^{2} T}{3 \pi\left(e^{\beta m_{e}}+1\right)}\left[\frac{1}{\epsilon}-2 \ln \frac{m_{D}}{\mu}+\frac{5}{3}+\ln (4 \pi)\right. \\
& -\gamma-2 \ln 2]\}\left\langle r^{2}\right\rangle_{n}+O\left(\alpha r^{2} E m_{D}^{2}\right) .
\end{aligned}
$$

We see that indeed the preceding UV divergences cancel those of Eq. (22), as expected. There is a subtle point in this calculation, however, which we discuss in Appendix E, which must be correctly dealt with in order to get the UV divergence of the last line [that cancels the IR divergence in the second to last line of Eq. (22)]. As in the massless case, the contribution from scale $m_{D}$ can be encoded in a correction to the potential, and this summed to $\delta V_{r}$,

$$
\begin{aligned}
& \delta V=-\frac{4 \alpha^{2} f\left(m_{e} \beta\right) m_{e}^{2} r}{\pi}-\frac{2 \alpha^{2}}{\pi r} \int_{0}^{\infty} \frac{d u}{\sqrt{u^{2}+1}\left(e^{\beta m_{e} \sqrt{u^{2}+1}}+1\right)}[1-\cos (\sigma u)-\sigma u \operatorname{Si}(\sigma u)] \\
& +\frac{\alpha^{2}}{3 \pi r} \int_{0}^{\infty} \frac{d u \sqrt{u^{2}+1}}{u^{2}\left(e^{\beta m_{e} \sqrt{u^{2}+1}}+1\right)}\left[2-3 \sigma^{2} u^{2}+\left(\sigma^{2} u^{2}-2\right) \cos (\sigma u)+\sigma u \sin (\sigma u)+\sigma^{3} u^{3} \operatorname{Si}(\sigma u)\right] \\
& -\frac{\alpha}{\pi} T m_{D}^{2} r^{2}\left(\beta m_{e}\right)^{3} \int_{0}^{\infty} d u \frac{u \sqrt{u^{2}+1}}{e^{\beta m_{e} \sqrt{u^{2}+1}}+1}+\frac{\alpha m_{D}^{3} r^{2}}{6}-\frac{i 16 \alpha^{2} T^{3} g\left(m_{e} \beta\right) r^{2}}{3 \pi}\left[-\ln \left(r m_{D}\right)+\frac{4}{3}-\gamma\right] \\
& -\frac{i 4 \alpha^{2} T}{\pi\left(e^{\beta m_{e}}+1\right)}\left[\frac{1}{2}-\ln (r T)-\ln 2-\left(e^{\beta m_{e}}+1\right) \int_{0}^{\infty} \frac{d u}{u\left(e^{\beta m_{e} \sqrt{u^{2}+1}}+1\right)}+\int_{0}^{\infty} \frac{d u e^{-\beta m_{e} u}}{u}\right] \\
& +\frac{i 4 \alpha^{2}}{\pi r} \int_{0}^{\infty} \frac{d u}{u^{4}} \sin (\sigma u)\left[L i_{2}\left(-e^{\beta m_{e} \sqrt{u^{2}+1}}\right)+\beta m_{e} \sqrt{u^{2}+1} \ln \left(1+e^{\beta m_{e} \sqrt{u^{2}+1}}\right)\right. \\
& \left.+\frac{\pi^{2}}{6}-\frac{\left(\beta m_{e}\right)^{2}}{2}\left(u^{2}+1\right)-g\left(m_{e} \beta\right)+\frac{\left(\beta m_{e}\right)^{2} u^{2}}{2\left(e^{\beta m_{e}}+1\right)}\right]+\frac{i 4 \alpha^{2} T}{\pi} \int_{0}^{\infty} \frac{d u}{u^{3}}\left[\frac{\operatorname{Sinc}(\sigma u)-1}{e^{\beta m_{e} \sqrt{u^{2}+1}}+1}-\frac{\operatorname{Sinc}(\sigma u)-e^{-8 \beta^{3} m_{e}^{3} u^{3}}}{e^{\beta m_{e}}+1}\right] \\
& -\frac{i 8 \alpha^{2} m_{e}^{2} T r^{2}}{3 \pi\left(e^{\beta m_{e}}+1\right)}\left[-\ln \left(r m_{D}\right)+\frac{4}{3}-\gamma\right]-\frac{i 16 \alpha^{2} m_{e}^{2}}{3 \pi T\left(e^{\beta m_{e}}+1\right)} \Gamma(-2 / 3)+O\left(\alpha^{3} T\right) \text {. }
\end{aligned}
$$

The energy shift and the decay width at first order in perturbation theory can be obtained from Eq. (19).

$$
\text { 3. } T \gg p
$$

This case is very similar to the $m_{e}=0$ one. We start with NRQED and integrate out scale $T$ first. Since $T \gg m_{e}$, the mass-dependent HTL propagators may be expanded in $m_{e} / T$, and hence become the usual HTL propagators with NLO contributions due to the nonvanishing electron mass. Hence, the finite-mass effects do not affect the gross features of the system. In particular, in the $e T \sim p$ case, the dissociation temperature will be similar to the one in the massless case. For $e T \gg p$, like in the massless case, no bound state is expected to survive.

\section{B. Higher-energy states $(n \geqslant 3)$}

As mentioned before, these states fulfill $m_{e} \gg p \gg E$, and hence relativistic electron-positron pairs may be integrated out before the momentum transfer $p$ is. In Secs. III B 1 and III B 2, let us see how this is carried out depending on what the temperature is.

\section{1. $m_{e} \gg T$}

In this case, we can start with an NRQED Lagrangian for the muon in which the relativistic electron-positron pairs have already been integrated out, which gives rise to $1 / m_{e}^{2}$ corrections to the Maxwell Lagrangian. Since there are neither electrons nor positrons in the thermal bath, the different situations coincide with those of the hydrogen atom, and the relevant expressions can be read off Secs. III and IV of Ref. [1] by making $m \rightarrow m_{\mu}$ (up to $1 / m_{e}^{2}$ corrections).

$$
\text { 2. } m_{e} \lesssim T
$$

In this case, we can start with an NRQED Lagrangian for the muon keeping the relativistic Dirac Lagrangian for the electron in it. When $m_{e} \sim T$, both scales must be integrated out 
at the same time. In the photon sector, a mass-dependent HTL Lagrangian is induced (see Sec. V A 1 of Ref. [1]). In the electron and positron sectors, a temperature-dependent NRQED ${ }_{T}$ Lagrangian for each particle is induced (see Sec. VA 1 of Ref. [1]). In the muon sector, a temperature-dependent $\mathrm{NRQED}_{>T}$ Lagrangian is also induced. At lower orders, it can be obtained from the diagrams of Sec. IV of Ref. [1], together with diagrams containing an electron-positron loop. The most important effect is the appearance of two mass shifts, one $\sim \alpha T^{2} / m_{\mu}$ from diagram (38) of Ref. [1] and the other one $\sim \alpha^{2} m_{e}$ from the second diagram in Fig. 4. In the proton sector, an analogous mass shift $\sim \alpha^{2} m_{e}$ occurs, which is due to a diagram obtained from the previous one by changing the muon line with a proton line.

The next step is to integrate out scale $p$, namely, matching to pNRQED using the HTL Lagrangian. This has already been done in Sec. VB of Ref. [1]. At LO, this produces a temperature-dependent potential and further mass shifts, which can be read from a corrected version of formulas (58) and (60) in that reference (making $m \rightarrow m_{e}$ ). The origin of the corrections is discussed in Appendix E and boils down to a simple replacement; see Eq. (E17). Both the potential and the mass shift contain an imaginary part, which becomes more important than the real part starting at some temperature $T_{d}$, which we call dissociation temperature. In Sec. III C, the dissociation temperatures will be estimated for several states.

For $T \gg m_{e}$, none of the higher-energy states exists anymore, since the dissociation temperatures fulfill $m_{e}>T$, see Sec. III C.

\section{Dissociation temperatures, level shifts, and decay widths}

The dissociation temperatures will be estimated in a similar way as they were in the hydrogen atom case [1]. This is by identifying the momentum scale for which the real and imaginary parts of the momentum space potential are equal, $p \sim(16 \alpha)^{1 / 3}\left[g\left(m_{e} \beta\right)+\left(m_{e} \beta\right)^{2} n_{F}\left(m_{e} \beta\right) / 2\right]^{1 / 3} T=: m_{d}$ and equating it to the typical momentum transfer in the muonic hydrogen atom ${ }^{2} p \sim m_{\mu} \alpha / n^{2}$. The results are displayed in Table I. Table II shows the same results for the hydrogen atom. In order to carry out the estimates, we also have used the formulas of Appendix E, which are meant for higher excited states $\left(T \sim m_{e} \gg p\right)$, for the case of the lower-lying states, instead of the $m_{e}=0$ formulas. This is indeed legitimated: We are just not taking advantage of the fact that $T_{d} \gg m_{e}$ for these states, which produces the simplifications discussed in Sec. III A 3 .

\footnotetext{
${ }^{2}$ Note that in Ref. [1], the typical momentum of the electron $p \sim m_{e} \alpha / n$ was used rather than the typical momentum transfer $p \sim m_{e} \alpha / n^{2}$, as we have adopted here. For the lower-lying states, the order of magnitude estimates does not differ much, but for higher-energy, estimates may differ considerably. We have also identified an error, which affects the dissociation temperatures, in the computation of the potential (57) of Ref. [1], which is explained and corrected in Appendix E. Here, we reproduce Table I of Ref. [1] in Table II with the current choice of $p$ and the correct version of the potential for the sake of comparison.
}

TABLE I. Dissociation temperature for the lower-lying states of muonic hydrogen.

\begin{tabular}{cccc}
\hline \hline$n$ & $T_{d}(\mathrm{MeV})$ & $m_{D}(\mathrm{MeV})$ & $m_{d}(\mathrm{MeV})$ \\
\hline 1 & 1.7 & 0.16 & 0.77 \\
2 & 0.41 & 0.036 & 0.19 \\
3 & 0.19 & 0.012 & 0.086 \\
4 & 0.13 & 0.0056 & 0.048 \\
5 & 0.10 & 0.0030 & 0.031 \\
\hline \hline
\end{tabular}

From the results in Table I, we see that only the lowest-lying states $n=1,2$ survive at temperatures of the electron-positron plasma $m_{e} \lesssim T$. Hence, only transitions between these levels might be observed in an eventual experiment. We will focus on the experimentally prominent $K \alpha$ transition [17]. We display our results for the energies of the $1 S$ and $2 P$ states, and for the energy of the $K \alpha$ transition as a function of temperature in the range $T \in(2,0.05) \mathrm{MeV}$ in Figs. 6-8, respectively. The calculations have been carried out numerically, using firstorder perturbation theory for the potential (27).

Another observable that we can predict with our results is the decay width. In Fig. 9, we show the decay width for the $1 S$ state as a function of the temperature. By comparing Figs. 6 and 9 , it can be seen that $T_{d} \sim 1.7 \mathrm{MeV}$ is the temperature that makes the decay width of the same magnitude as the binding energy.

\section{DISCUSSION AND CONCLUSIONS}

In this paper, we have discussed the properties of muonic hydrogen in a thermal bath, which may consist not only of blackbody radiation, but also of an electron-positron plasma. We have further developed the effective theory techniques for bound-state systems at finite temperature initiated in Ref. [1], in particular, the application of DR to the factorization of the various scales in the system. They enormously facilitate the organization of the calculation. For instance, they show when Coulomb or HTL resummations are necessary and when they are not. In addition, both partial and final results are naturally obtained as a series of small scales over large ones, thus providing good control on the systematics.

We discussed two cases. First, we addressed the academic case of muonic hydrogen with a vanishing electron mass, which turns out to be closer to heavy quarkonium states than the actual case with a nonvanishing electron mass, which we addressed next. All the thermal modifications we found turned out to be spin independent.

TABLE II. Dissociation temperature for the lower-lying states of hydrogen.

\begin{tabular}{lcll}
\hline \hline$n$ & $T_{d}(\mathrm{keV})$ & $m_{D}(\mathrm{keV})$ & $m_{d}(\mathrm{keV})$ \\
\hline 1 & 49 & 0.15 & 3.7 \\
2 & 36 & 0.020 & 0.93 \\
3 & 31 & 0.0061 & 0.41 \\
4 & 28 & 0.0025 & 0.23 \\
5 & 26 & 0.0013 & 0.15 \\
\hline \hline
\end{tabular}




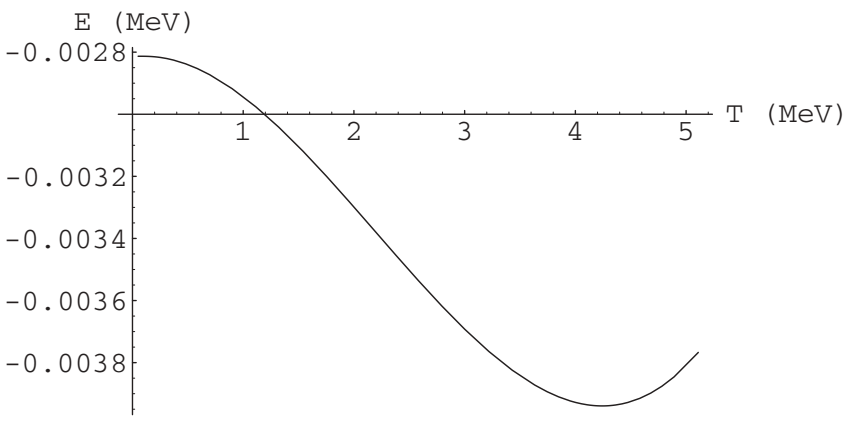

FIG. 6. $E$ vs $T$ for the $1 S$ state. This result was computed using the assumption that $\frac{1}{r} \gg m_{D}$, so we expect important deviations from the real energy starting at $T \sim 4 \mathrm{MeV}$.

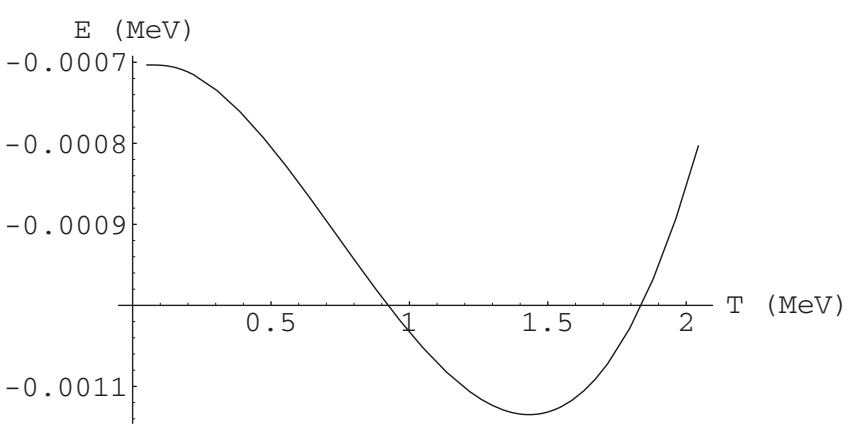

FIG. 7. $E$ vs $T$ for the $2 P$ state. For the $2 P$ state, the typical radius is four times larger than for the $1 S$ state, so we expect important deviations from the real energy starting at $T \sim 1 \mathrm{MeV}$.

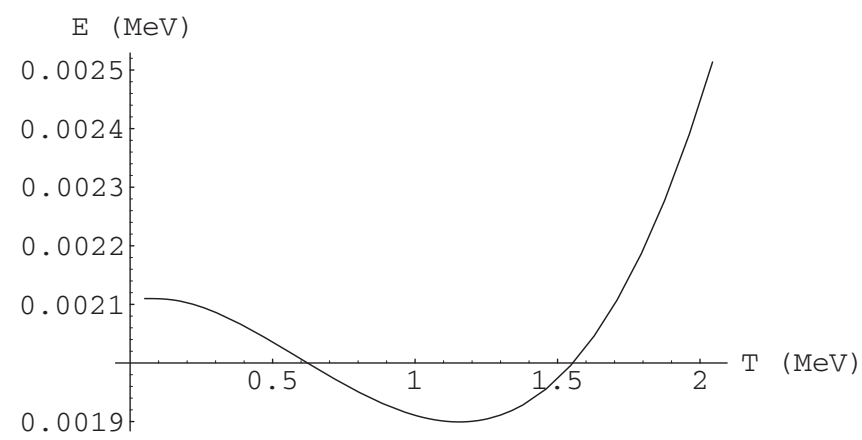

FIG. 8. $K_{\alpha}$ transition vs $T$. This result is reliable until $T \sim$ $1 \mathrm{MeV}$. Note that this temperature is twice what we predicted for the dissociation temperature of the $2 P$ state.

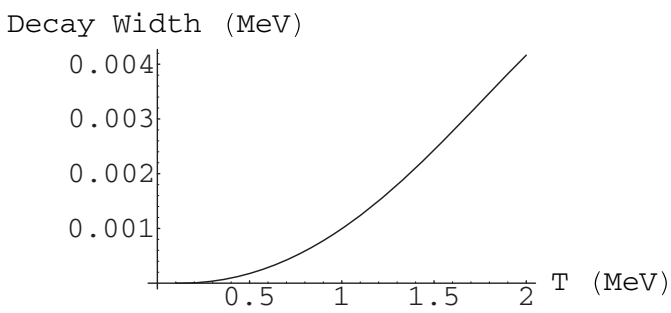

FIG. 9. Decay width for the $1 S$ state. As for the binding energy, this has been computed using the assumption that $\frac{1}{r} \gg m_{D}$.
TABLE III. Dissociation temperature for $\Upsilon(1 S)$ for different values of the charm mass. The $n_{f}=3$ light quark masses are set to zero. We use the values of the Bohr radius and $\Lambda_{\mathrm{QCD}}$ found in Table 2.1 of Ref. [42] as an input. The values of these parameters for $n_{F}=3$ are used for all values of $m_{c}$ except for $m_{c}=0$, where we use the ones for $n_{F}=4$.

\begin{tabular}{cc}
\hline \hline$m_{c}(\mathrm{MeV})$ & $T_{d}(\mathrm{MeV})$ \\
\hline$\infty$ & 480 \\
5000 & 480 \\
2500 & 460 \\
1200 & 440 \\
0 & 420 \\
\hline \hline
\end{tabular}

In the zero electron mass case, we studied how the effects of vacuum polarization modify the picture that we encountered in normal hydrogen [1]. The modifications turned out to be important when the temperature was higher than the binding energy. For instance, they gave the LO contribution to a hypothetical $K \alpha$ transition for high enough temperatures Eq. (2). For temperatures below dissociation, we presented the LO and selected NLO thermal corrections to the binding energy and decay width.

In the actual electron mass case, muonic hydrogen behaves very much the same as hydrogen for temperatures below the electron mass. For temperatures higher or on the order of the electron mass, the vacuum polarization effects are sizable, and, at some point, make the bound states dissociate. In Table I, we display the dissociation temperature for the lower-lying states. We also calculated the thermal modifications to a number of observables before dissociation occurred. For instance, we plotted the dependence of the $K \alpha$ transition on temperature in Fig. 8, which could be tested experimentally in the future [8].

We close with a concrete application to the heavy quarkonium case. As we have mentioned before, the way a finiteelectron mass affects muonic hydrogen is similar to the way a finite-charm quark mass affects bottomonium [41]. Since this should also be the case at finite temperature, we can easily translate the results for the dissociation temperature of muonic hydrogen to the QCD case, which we show in Table III.

\section{ACKNOWLEDGMENTS}

We acknowledge financial support from the RTN Flavianet MRTN-CT-2006-035482 (EU), the FPA2007-60275/MEC grant (Spain), and the 2009SGR502 CUR grant (Catalonia). J.S also acknowledges financial support form the ECRI HadronPhysics2 (Grant Agreement No. 227431) (EU), the FPA2007-66665-C02-01/MEC grant, and the Consolider Ingenio program CPAN CSD2007-00042 (Spain). M.A.E. has also been supported by a MEC FPU (Spain).

\section{APPENDIX A: NOTATION FOR THE DIFFERENT EFFECTIVE FIELD THEORIES}

At zero temperature, there are three different energy scales for nonrelativistic bound states. These are the hard scale (for muonic hydrogen $m_{\mu}$ ), the soft scale $m_{\mu} \alpha$, and the ultrasoft scale $m_{\mu} \alpha^{2}$. Moreover, a finite-temperature system also has a 
different energy scale as $T, e T, e^{2} T, \ldots$ This makes it hard to find a comprehensible notation for all the effective field theories that may arise from integrating out the different degrees of freedom. In this paper, we have used the following notation. Basically, we named the effective field theories as one would do for zero temperature, and we encoded the temperature information in a subindex. Subindex $T$ means that the temperature has been integrated out, also subindex $m_{D}$ means that scale $e T$ has been integrated out. Since the matching coefficients of the effective field theory that we obtain after integrating out $m_{\mu}(p)$ and $T$ is not the same if $m_{\mu}(p) \sim T$ or if $m_{\mu}(p) \gg T$, we include a symbol $<,>$, or a blank depending on the relation between these scales. For example, if we are in $m_{\mu}(p) \sim T$, we will arrive at $\mathrm{NRQED}_{T}\left(\mathrm{pNRQED}_{T}\right)$, but if we are in $m_{\mu}(p) \gg T$, we reach NRQED ${ }_{>T}\left(\mathrm{pNRQED}_{>T}\right)$ because $T$ is smaller than the energy cutoff of NRQED (pNRQED).

\section{APPENDIX B: BASIC FORMULAS}

In this appendix, we display a number of formulas of the real-time formalism that are relevant to the paper. Our notation closely follows Ref. [31]. Recall that, in this formalism, there is a doubling of degrees of freedom [30]. Fields are labeled 1 or 2 . Fields 1 (2) only interact with fields 1 (2) according to (minus) the original interaction Lagrangian. Fields 1 may be converted to fields 2 , and vice versa, through propagation so that propagators become $2 \times 2$ matrices. For instance, for a free scalar field, we have

$$
\begin{aligned}
\Delta(K)= & \left(\begin{array}{cc}
\frac{1}{K^{2}-m^{2}+i \epsilon} & 0 \\
0 & \frac{-1}{K^{2}-m^{2}-i \epsilon}
\end{array}\right)-2 \pi i \delta\left(K^{2}-m^{2}\right) \\
& \times\left[\begin{array}{cc}
n_{B}\left(k_{0}\right) & \theta\left(-k_{0}\right)+n_{B}\left(k_{0}\right) \\
\theta\left(k_{0}\right)+n_{B}\left(k_{0}\right) & n_{B}\left(k_{0}\right)
\end{array}\right],
\end{aligned}
$$

where $i \Delta(K)$ is the Feynman propagator, and $n_{B}\left(k_{0}\right)$ is the Bose distribution function.

For the tree-level propagator of the transverse electromagnetic field in the Coulomb gauge, $i \Delta_{i j}(K)$, we have

$$
\Delta_{i j}(K)=\left(\delta_{i j}-\frac{k_{i} k_{j}}{|\mathbf{k}|^{2}}\right) \Delta(K)
$$

The tree-level $A_{0}$-propagator matrix in the Coulomb gauge is diagonal, traceless, and the 11 component coincides with the propagator at zero temperature.

For the tree-level propagator of a Dirac fermion field $i S(K)$, we have $S(K) \equiv(K K+m) \tilde{\Delta}(K)$, where $\tilde{\Delta}(K)$ follows from $\Delta(K)$ by replacing $n_{B}$ by $-n_{F}, n_{F}$ being the Fermi-Dirac distribution.

Since we are always in case $m_{\mu} \gg T$, all vertices involving muons or protons will be type 1 . However, vertices involving photons and electrons can be both type 1 and type 2. At the order we are calculating, it turns out that we only need propagators of type 11 for the photons, either at tree level or including one-loop self-energies.

For computations that require loop-corrected propagators (for example, Figs. 2 and 3), it is convenient to use the so-called Keldysh representation [31]. In this representation, the retarded, advanced, and symmetric propagators are defined as

$$
\begin{aligned}
& \Delta_{R}=\Delta_{11}-\Delta_{12}, \\
& \Delta_{A}=\Delta_{11}-\Delta_{21}, \\
& \Delta_{S}=\Delta_{11}+\Delta_{22} .
\end{aligned}
$$

Notice from Eq. (B1) that at tree level only, $\Delta_{S}$ depends on the temperature.

In order to calculate loop corrections to the $\Delta_{11}$ propagator in an efficient way, we use the following method [31]:

(1) We compute the $\Delta_{R}$ propagator, using the fact that, for this propagator, the Dyson equation is of the zero-temperature type (this is not so for $\Delta_{S}$ ),

$$
\Delta_{R}=\Delta_{R}^{0}+\Delta_{R}^{0} \Pi_{R} \Delta_{R},
$$

where the self-energy $\Pi_{R}$ is

$$
\Pi_{R}=\Pi_{11}+\Pi_{12},
$$

and $\Delta_{R}^{0}$ is the tree-level retarded propagator obtained according to formulas (B1)-(B3).

(2) The advanced propagator $\Delta_{A}$ is the complex conjugate of the retarded one, and the symmetric propagator $\Delta_{S}$ reads, in the bosonic case,

$$
\Delta_{S}(K)=\left[1+2 n_{B}\left(\left|k_{0}\right|\right)\right] \operatorname{sgn}\left(k_{0}\right)\left[\Delta_{R}(K)-\Delta_{A}(K)\right] .
$$

(3) Finally, one obtains

$$
\Delta_{11}=\frac{1}{2}\left(\Delta_{R}+\Delta_{A}+\Delta_{S}\right) .
$$

Note that, for $\left|k_{0}\right| \ll T$, the size of the symmetric propagator for bosons is larger than what one would expect from naive power counting,

$$
\Delta_{S}(K)=\frac{T}{k_{0}}\left[\Delta_{R}(K)-\Delta_{A}(K)\right]
$$

plus terms suppressed by $1 / T$. This effect is called Bose enhancement, and it complicates the power counting of the EFTs at scales lower than the temperature.

\section{APPENDIX C: CALCULATIONS IN SEC. II A}

What all these computations have in common is that the starting point is pNRQED. In this effective theory and in all the theories that are derived from it by integrating out further degrees of freedom, the leading correction to the Hamiltonian is $[6,28]$

$$
\delta H=i e^{2} r^{i} \int \frac{d^{D} k}{(2 \pi)^{D}} \frac{1}{E-H-k_{0}+i \epsilon}\left(k_{0}^{2} \Delta_{i j}+k_{i} k_{j} \Delta_{00}\right) r^{j},
$$

with $\Delta_{i j}$ and $\Delta_{00}$ being the 11 transverse and $A_{0}$ photon propagators, respectively. $\delta H$ is a potential $\delta V$ (energy independent) only if $E$ is much smaller than the scales inside the $\Delta$ propagators. Depending on the concrete calculation that one is doing, the explicit expression for $\Delta$ may be different.

\section{Integrating out the $T$ scale}

After integrating out scale $T$, we will reach $\mathrm{pNRQED}_{>T}$. At LO, the difference between $\mathrm{pNRQED}$ and $\mathrm{pNRQED}_{>T}$ will 
be a correction on the potential of the type of Eq. (C1) where the internal momentum $K$ is of order $T$.

The LO correction (2) is obtained using the tree-level photon propagator in Eq. (C1). If one uses the Coulomb gauge, thermal effects only appear in transverse photons. This computation is done in detail for different relations between $T$ and $E$ in Ref. [1],

$$
\begin{aligned}
\delta V^{\mathrm{LO}}= & e^{2} r^{i} \int \frac{d^{D} k}{(2 \pi)^{D}} \frac{k_{0}^{2}}{E-H-k_{0}+i \epsilon} \\
& \times\left(\delta_{i j}-\frac{k_{i} k_{j}}{|\mathbf{k}|^{2}}\right) 2 \pi \delta\left(K^{2}\right) n_{B}\left(k_{0}\right) r^{j} .
\end{aligned}
$$

The NLO potential comes from including one-loop corrections with the photon propagator (this corresponds to the diagram of Fig. 3). This can be done analytically for $T \gg E$. The required expressions for the photon propagator are found in Sec. III B of Ref. [6]. Since $T \gg E$, the muonic hydrogen propagator can be expanded

$$
\frac{1}{E-H-k_{0}+i \epsilon} \rightarrow \frac{1}{-k_{0}+i \epsilon}-\frac{(E-H)}{\left(-k_{0}+i \epsilon\right)^{2}}+\cdots .
$$

Let us compute the first term in this expansion,

$$
\begin{aligned}
\delta V^{\mathrm{NLO} a}= & i e^{2} r^{i} \int \frac{d^{D} k}{(2 \pi)^{D}} \frac{1}{-k_{0}+i \epsilon} \\
& \times\left(k_{0}^{2} \Delta_{i j}^{1-\text { loop }}+k_{i} k_{j} \Delta_{00}^{1-\text { loop }}\right) r^{j} .
\end{aligned}
$$

Looking at the expressions of Ref. [6] and using the relation (B9), one sees that both $\Delta_{i j}$ and $\Delta_{00}$ are even functions in $k_{0}$, so one can use for the first term of the expansion,

$$
\frac{i}{-k_{0}+i \epsilon} \rightarrow \pi \delta\left(-k_{0}\right) \text {. }
$$

So, we only need the photon propagator in the limit $k_{0} \rightarrow 0$ to get the LO.

In Ref. [6], the propagators are given as an integral of a parameter $q_{0}$. The best strategy to perform the calculation is to integrate first over internal momentum and leave the integration of this parameter for the end. This computation was done in Sec. IV B 1 of Ref. [6], here we take the Abelian limit making $C_{F}=1$ and $C_{A}=0$,

$$
\begin{aligned}
\delta V^{\mathrm{NLO} a}= & \frac{3}{2} \zeta(3) \frac{\alpha}{\pi} r^{2} T m_{D}^{2}+i \frac{\alpha}{6} r^{2} T m_{D}^{2}\left[\frac{1}{\epsilon}+\gamma_{E}+\ln \pi\right. \\
& \left.-\ln \frac{T^{2}}{\mu^{2}}+\frac{2}{3}-4 \ln 2-2 \frac{\zeta^{\prime}(2)}{\zeta(2)}\right] .
\end{aligned}
$$

For the NLO in Eq. (3), namely, the contributions coming from second term in Eq. (C3), we will restrict ourselves to the extraction of the leading IR logarithmic behavior. We use the fact that, in the IR, the photon self-energy approaches the HTL limit, so instead, we substitute the complete self-energy by $\Pi_{\mathrm{HTL}} e^{-\beta^{2} k^{2}}$. The factor $e^{-\beta^{2} k^{2}}$ is introduced to regulate $\mathrm{UV}$ divergences and does not affect the leading IR behavior we are interested in. The HTL self-energy has the property that can be written as $\Pi_{\mathrm{HTL}}=m_{D}^{2} f\left(k_{0} / k\right)$ with $f(x)$ as a nontrivial function. Performing the change of variables $k_{0}=k x$, the IR behavior can easily be extracted. First, we compute the contribution from the retarded part of the longitudinal photon propagator,

$$
\begin{aligned}
\delta V_{L}^{\mathrm{NLO} b} & =i e^{2} r^{i}(E-H) \int \frac{d^{D} k}{(2 \pi)^{D}} \frac{k_{i} k_{j}}{k^{4}} \frac{\Pi_{R}^{L}\left(k_{0}, k\right)}{\left(k_{0}-i \epsilon\right)^{2}} r^{j} \\
& =-\frac{e^{2}}{2 m_{\mu}} \int \frac{d^{D} k}{(2 \pi)^{D}} \frac{\Pi_{R}^{L}\left(k_{0}, k\right)}{k^{2}\left(k_{0}-i \epsilon\right)^{2}} .
\end{aligned}
$$

We have used that $r^{i}(E-H) r_{i}=-\frac{1}{2 m_{\mu}}$ plus terms that vanish on the physical state. As we are only interested in the leading logarithm behavior, the change $\Pi_{R}^{L}\left(k_{0}, k\right) \rightarrow$ $\Pi_{\mathrm{HTL}, R}^{L}\left(k_{0}, k\right) e^{-\beta^{2} k^{2}}=m_{D}^{2} f_{R}^{L}\left(k_{0} / k\right) e^{-\beta^{2} k^{2}} \quad$ can be made, where

$$
\begin{gathered}
f_{R}^{L}(x)=1-\frac{x}{2} \ln \left(\frac{x+1+i \epsilon}{x-1+i \epsilon}\right), \\
\delta V_{L}^{\mathrm{NLO} b}=-\frac{i e^{2} m_{D}^{2}}{2 m_{\mu}} \int \frac{d^{D} k}{(2 \pi)^{D}} \frac{f_{R}^{L}\left(k_{0} / k\right) e^{-\beta^{2} k^{2}}}{k^{2}\left(k_{0}-i \epsilon\right)^{2}} .
\end{gathered}
$$

The preceding equality is only true as far as the leading IR behavior is concerned. Now, with the change $k_{0}=k x$,

$$
\begin{aligned}
\delta V_{L}^{\mathrm{NLO} b}= & -\frac{i e^{2} m_{D}^{2}}{2 m_{\mu}} \frac{\Omega_{D-1}}{(2 \pi)^{D}} \int_{0}^{\infty} d k k^{D-5} e^{-\beta^{2} k^{2}} \int_{-\infty}^{\infty} \frac{d x f_{R}^{L}(x)}{(x-i \epsilon)^{2}} \\
= & -\frac{i e^{2} m_{D}^{2}}{4 m_{\mu}} \frac{\Omega_{D-1}}{(2 \pi)^{D}}\left(\frac{T}{\mu}\right)^{D-4} \Gamma\left(\frac{D-4}{2}\right) \\
& \times \int_{-\infty}^{\infty} \frac{d x f_{R}^{L}(x)}{(x-i \epsilon)^{2}} .
\end{aligned}
$$

$\Omega_{D-1}$ is the $D-1$-dimensional solid angle. Note that the contribution from the advanced part of the longitudinal photon propagator is obtained by replacing $f_{R}^{L}(x) \rightarrow\left(f_{R}^{L}\right)^{*}(x)$ in Eq. (C10). Since it has all the singularities in the upper half complex plane, the corresponding integral gives zero. The integral over $x$ in Eq. (C10) can be done using standard techniques from integration in the complex plane, and the result is

$$
\int_{-\infty}^{\infty} \frac{d x f_{R}^{L}(x)}{(x-i \epsilon)^{2}}=-\pi^{2} .
$$

The contribution from the symmetric part of the longitudinal photon propagator does not produce IR divergences in DR. So, we do not need to compute it here, as we are only interested in the logarithms.

Next, we proceed analogously for the transverse photon propagator. As in the longitudinal part, only the retarded plus advanced contribution is IR sensible. We approximate $\Pi_{R}^{T}\left(k_{0}, k\right)=m_{D}^{2} f_{R}^{T}\left(k_{0} / k\right) e^{-\beta^{2} k^{2}}$ where now,

$$
f_{R}^{T}(x)=\frac{1}{2}\left[x^{2}-\left(x^{2}-1\right) \frac{x}{2} \ln \left(\frac{x+1+i \epsilon}{x-1+i \epsilon}\right)\right],
$$

then

$$
\begin{aligned}
\delta V_{T}^{\mathrm{NLO} b}= & -\frac{i e^{2} r^{i}(E-H) r^{j}}{2} \int \frac{d^{D} k}{(2 \pi)^{D}} m_{D}^{2} e^{-\beta^{2} k^{2}} \\
& \times\left(\delta_{i j}-\frac{k_{i} k_{j}}{k^{2}}\right)\left[\frac{f_{R}^{T}\left(k_{0} / k\right)}{\left[k_{0}^{2}-k^{2}+i \operatorname{sgn}\left(k_{0}\right) \epsilon\right]^{2}}\right. \\
& \left.+\frac{f_{A}\left(k_{0} / k\right)}{\left[\left(k_{0}^{2}-k^{2}-i \operatorname{sgn}\left(k_{0}\right) \epsilon\right]^{2}\right.}\right] .
\end{aligned}
$$


The first (second) terms in the square brackets have all the singularities in the lower (upper) complex $k_{0}$ half plane, and hence, the whole expression vanishes. The contribution from the symmetric piece of the propagator also vanishes for the same reason. Hence,

$$
\delta V^{\mathrm{NLO}}=0
$$

\section{The $E$ scale for $T \gg E \gg m_{D}$}

In order to obtain Eqs. (6) and (7), the starting point is $\mathrm{pNRQED}_{T}$. In this effective theory, the atom self-energy gives the correction for the Hamiltonian (C1). Recall that the photon propagators in Eq. (C1) must be taken in the HTL approximation. We use the method of integration by regions $[36,37]$ in order to evaluate it. In this integral, there are three relevant regions: $k, k_{0} \sim E$ with $\lambda=k_{0}-k \sim E$ (we call it the off-shell region), $k, k_{0} \sim E$ with $\lambda \sim m_{D}$ (collinear region), and $k, k_{0} \sim m_{D}\left(m_{D}\right.$ region). By power counting, one can see that the $m_{D}$ region will just be a higher-order correction, so we focus on the regions $k, k_{0} \sim E$. In the off-shell region, the atom propagator cannot be expanded, but the HTL photon propagator can. For example, the longitudinal photon retarded propagator can be written as

$$
\Delta_{00, R}=\frac{1}{k^{2}+m_{D}^{2} f_{R}^{L}\left(k_{0} / k\right)} .
$$

After the compulsory expansion,

$$
\Delta_{00, R}=\frac{1}{k^{2}}-\frac{m_{D}^{2} f_{R}^{L}\left(k_{0} / k\right)}{k^{4}}+\cdots .
$$

The fact that the nontrivial functions appear only in the numerator after the expansion is crucial in order to be able to do the integration analytically. The collinear region does not contribute in the part that is related to longitudinal photons.

The transverse photon retarded propagator is

$$
\Delta_{i j, R}=\frac{\left(\delta_{i j}-\frac{k_{i} k_{j}}{k^{2}}\right)}{k_{0}^{2}-k^{2}-m_{D}^{2} f_{R}^{T}\left(k_{0} / k\right)+i \epsilon} .
$$

In the off-shell region, an expansion, such as the one for the longitudinal photon propagator has to be made. Also, in the collinear region (that has $k, k_{0} \sim E$ but $k_{0}^{2}-k^{2} \sim m_{D}^{2}$ ), the atom propagator cannot be expanded, but the HTL photon propagator has to be expanded around the region $\left|k_{0} / k\right| \sim 1$. As in the previous case, this expansion makes it possible to proceed analytically.

Details of this computation can be found in Ref. [38].

\section{Integrating out $m_{D}$ for $m_{D} \gg E$}

After integrating out $m_{D}$, we will arrive at what we will call $\mathrm{pNRQED}_{>m_{D}}$. In the photon sector, we will have a nontrivial action, but we will not need it at the level of precision we are working. In the atom sector, a correction of the potential in the matching between $\mathrm{pNRQED}_{T}$ (or $\mathrm{pNRQED}_{>T}$ ) and $\mathrm{pNRQED}_{>m_{D}}$ of the form of Eq. (C1) appears where the internal momentum is of order $k \sim m_{D}$.
Because $m_{D} \gg E$, we can also put $E-H=0$ at LO in the atom propagator as in Sec. C 1. Hence, we will need the HTL photon propagator in the $k_{0} \rightarrow 0$ limit (i.e., $T \gg k \gg k_{0}$ ). The HTL photon propagators are very well known and can be found in Refs. [6,30,31]. In our case, the fact that $k_{0} \rightarrow 0$ makes

$$
\begin{gathered}
k_{0}^{2} \Delta_{i j}(K)=0, \\
k_{i} k_{j} \Delta_{00}(K)=k_{i} k_{j}\left[\frac{1}{k^{2}+m_{D}^{2}}-\frac{i \pi T m_{D}^{2}}{k\left(k^{2}+m_{D}^{2}\right)^{2}}\right] .
\end{gathered}
$$

Using this in Eq. (C1), the results of Eqs. (10) and (11) are obtained.

For the NLO (12), the calculation is very similar to the one we have carried out for the $T$ scale. As in that case, we will focus on the logarithmic behavior, now in the UV. As an example, we again study the retarded part of the longitudinal photon propagator [note that consistency with Eq. (C14) ensures that there will not be a logarithmic contribution from transverse photons],

$$
\begin{aligned}
& \delta V_{L}^{\mathrm{NLO} m_{D}} \\
& =-i e^{2} r^{i} \int \frac{d^{D} k}{(2 \pi)^{D}} \frac{k_{i} k_{j}(E-H)}{\left(k_{0}-i \epsilon\right)^{2}} \frac{1}{k^{2}+m_{D}^{2} f_{R}^{L}\left(k_{0} / k\right)} r^{j} \\
& \left.\quad=\frac{i e^{2}}{2 m_{\mu}} \int \frac{d^{D} k}{(2 \pi)^{D}} \frac{k^{2}}{\left(k_{0}-i \epsilon\right)^{2}} \frac{1}{k^{2}+m_{D}^{2} f_{R}^{L}\left(k_{0} / k\right)}, \quad \text { C } 20\right)
\end{aligned}
$$

using that scaleless integrals in DR are 0 ,

$\delta V_{L}^{\mathrm{NLO} m_{D}}=-\frac{i e^{2} m_{D}^{2}}{2 m_{\mu}} \int \frac{d^{D} k}{(2 \pi)^{D}} \frac{f_{R}^{L}\left(k_{0} / k\right)}{\left(k_{0}-i \epsilon\right)^{2}} \frac{1}{k^{2}+m_{D}^{2} f_{R}^{L}\left(k_{0} / k\right)}$.

Now is when the change $k_{0}=k x$ becomes useful,

$$
\begin{aligned}
\delta V_{L}^{\mathrm{NLO} m_{D}} \\
=-\frac{i e^{2} m_{D}^{2}}{2 m_{\mu}} \frac{\Omega_{D-1}}{(2 \pi)^{D}} \int_{-\infty}^{\infty} \frac{d x f_{R}^{L}(x)}{(x-i \epsilon)^{2}} \int_{0}^{\infty} \frac{d k k^{D-3}}{k^{2}+m_{D}^{2} f_{R}^{L}(x)} \\
=\frac{i e^{2} m_{D}^{2}}{4 m_{\mu}} \frac{\Omega_{D-1}}{(2 \pi)^{D}} \pi \operatorname{cosec}\left(\frac{D \pi}{2}\right)\left(\frac{m_{D}}{\mu}\right)^{D-4} \\
\quad \times \int_{-\infty}^{\infty} \frac{d x\left[f_{R}^{L}(x)\right]^{D-3}}{(x-i \epsilon)^{2}} .
\end{aligned}
$$

If one is only interested in the logarithmic behavior, one can put $D-3=1$ in the $x$ integration and use Eq. (C11). Formula (12) is readily obtained from the previous expression.

\section{UV behavior for $E \sim m_{D}$}

Although we have not been able to obtain an analytic result for Eq. (C1) in this situation, its UV behavior can easily be isolated as follows:

$$
\begin{aligned}
\frac{1}{E-} & H-k_{0}+i \epsilon \\
= & {\left[\frac{1}{E-H-k_{0}+i \epsilon}-\frac{1}{-k_{0}+i \epsilon}+\frac{(E-H)}{\left(-k_{0}+i \epsilon\right)^{2}}\right] } \\
& +\frac{1}{-k_{0}+i \epsilon}-\frac{(E-H)}{\left(-k_{0}+i \epsilon\right)^{2}} .
\end{aligned}
$$


The piece in brackets on the right-hand side of the preceding equation leads to a UV finite expression when substituted in Eq. (C1). So, the UV divergences arise from the remaining terms on the right-hand side of this equation. In fact, the computation for these UV divergences is exactly the same as in case $m_{D} \gg E$, which we have carried in Sec. C 3 .

\section{APPENDIX D: CALCULATIONS IN SEC. III A}

\section{Correction to the Coulomb potential in $\mathrm{PNRQED}_{T}$}

In this section, we deal with the matching procedure that has to be done for $T \sim p$. In perturbation theory, the pNRQED potential is related to the Fourier transform of the longitudinal photon propagator in the limit where $p_{0} \rightarrow 0$ (for $P$ the external momentum of the propagator). For this temperature range, the propagator, which can be obtained by the procedure outlined in Appendix B, is needed for $T \sim m_{e} \sim p \gg p_{0}$. The retarded self-energy reads

$$
\begin{aligned}
\Pi_{R}(P)= & -\frac{2 e^{2}}{\pi^{2}} \int_{0}^{\infty} \frac{d k k^{2}}{\sqrt{k^{2}+m_{e}^{2}}\left(e^{\beta \sqrt{k^{2}+m_{e}^{2}}}+1\right)} \\
& +\frac{e^{2}}{\pi^{2} p} \int_{0}^{\infty} \frac{d k k\left(2 k^{2}+2 m_{e}^{2}-p^{2} / 2\right)}{\sqrt{k^{2}+m_{e}^{2}}\left(e^{\beta \sqrt{k^{2}+m_{e}^{2}}}+1\right)} \\
& \times \ln \left(\frac{|-p+2 k|}{|p+2 k|}\right)-\frac{2 i e^{2} p_{0}}{\pi p} \int_{p / 2}^{\infty} \frac{d k k}{e^{\beta \sqrt{k^{2}+m_{e}^{2}}}+1} \\
& -\frac{i e^{2} p_{0} m^{2}}{\pi p} \frac{1}{e^{\beta \sqrt{p^{2} / 4+m_{e}^{2}}}+1} .
\end{aligned}
$$

For $m_{e} \rightarrow 0$, this self-energy coincides with the Abelian limit of the one found in Ref. [6].

In order to obtain the potential, the first step is to use formulas (B8) and (B9) to get the corrections for the 11 propagator. Then, the propagator is related to potential plus the self-energy by the following formula:

$$
V(r)=-e^{2} \int \frac{d^{D-1} p}{(2 \pi)^{D-1}}\left(e^{i \mathbf{p} \cdot \mathbf{r}}-1\right) \Delta_{11}\left(p_{0}=0, p\right),
$$

at LO, this gives the Coulomb potential $V(r)=-\frac{\alpha}{r}$. For simplicity, we define

$$
V_{r}(r)=-e^{2} \int \frac{d^{D-1} p}{(2 \pi)^{D-1}} e^{i \mathbf{p} \cdot \mathbf{r}} \Delta_{11}\left(p_{0}=0, p\right),
$$

and

$$
V_{m}=-e^{2} \int \frac{d^{D-1} p}{(2 \pi)^{D-1}} \Delta_{11}\left(p_{0}=0, p\right)
$$

such that

$$
V(r)=V_{r}(r)-V_{m}
$$

For the NLO, we need the corrections to the propagator,

$$
\delta \Delta_{11}=\Delta_{1}+\Delta_{2}+\Delta_{3}+\Delta_{4}+\Delta_{5},
$$

where

$$
\begin{aligned}
& \Delta_{1}=-\frac{2 e^{2}}{\pi^{2} p^{4}} \int_{0}^{\infty} \frac{d k k^{2}}{\sqrt{k^{2}+m_{e}^{2}}\left(e^{\beta \sqrt{k^{2}+m_{e}^{2}}}+1\right)}, \\
& \Delta_{2}=\frac{2 e^{2}}{\pi^{2} p^{5}} \int_{0}^{\infty} \frac{d k k \sqrt{k^{2}+m_{e}^{2}}}{e^{\beta \sqrt{k^{2}+m_{e}^{2}}}+1} \ln \frac{|-p+2 k|}{|p+2 k|} \\
& =-\frac{2 e^{2}}{\pi^{2} p^{5}} \int_{0}^{\infty} \frac{d k k^{2} \sqrt{k^{2}+m_{e}^{2}}}{e^{\beta \sqrt{k^{2}+m_{e}^{2}}}+1} \\
& \times \int_{-1}^{1} d \lambda\left(\frac{1}{p-2 k \lambda+i \epsilon}+\frac{1}{p-2 k \lambda-i \epsilon}\right), \\
& \Delta_{3}=-\frac{e^{2}}{2 \pi^{2} p^{3}} \int_{0}^{\infty} \frac{d k k}{\sqrt{k^{2}+m_{e}^{2}}\left(e^{\beta \sqrt{k^{2}+m_{e}^{2}}}+1\right)} \ln \frac{|-p+2 k|}{|p+2 k|} \\
& =\frac{e^{2}}{2 \pi^{2} p^{3}} \int_{0}^{\infty} \frac{d k k^{2}}{\sqrt{k^{2}+m_{e}^{2}}\left(e^{\beta \sqrt{k^{2}+m_{e}^{2}}}+1\right)} \\
& \times \int_{-1}^{1} d \lambda\left(\frac{1}{p-2 k \lambda+i \epsilon}+\frac{1}{p-2 k \lambda-i \epsilon}\right), \\
& \Delta_{4}=-\frac{4 i T e^{2}}{\pi p^{5}} \int_{p / 2}^{\infty} \frac{d k k}{e^{\beta \sqrt{k^{2}+m_{e}^{2}}}+1}, \\
& \Delta_{5}=-\frac{2 i T e^{2} m_{e}^{2}}{\pi p^{5}} \frac{1}{e^{\beta \sqrt{p^{2} / 4+m_{e}^{2}}}+1} .
\end{aligned}
$$

The contribution of Eq. (D7) to Eq. (D2) leads to the first term in the first line of Eq. (22). In order to calculate the contribution of Eq. (D8) to Eq. (D2), it is convenient to leave the integration over the internal momentum $k$ to the end. Consider then,

$$
\begin{aligned}
& -\frac{e^{2}}{2} \int_{-1}^{1} d \lambda \int \frac{d^{D-1} p}{(2 \pi)^{D-1}} \frac{e^{i \mathbf{p} \cdot \mathbf{r}}}{p^{5}} \\
& \quad \times\left(\frac{1}{p-2 k \lambda+i \epsilon}+\frac{1}{p-2 k \lambda-i \epsilon}\right),
\end{aligned}
$$

and use

$$
\begin{aligned}
\frac{1}{p-2 k \lambda+i \epsilon} \\
=-\frac{1}{2 k \lambda-i \epsilon}-\frac{p}{(2 k \lambda-i \epsilon)^{2}}-\frac{p^{2}}{(2 k \lambda-i \epsilon)^{3}} \\
\quad-\frac{p^{3}}{(2 k \lambda-i \epsilon)^{4}}+\frac{p^{4}}{(2 k \lambda-i \epsilon)^{4}(p-2 k \lambda+i \epsilon)},
\end{aligned}
$$

in Eq. (D12),

$$
\begin{aligned}
& \frac{e^{2}}{2} \int_{-1}^{1} d \lambda\left[\frac{1}{(2 k \lambda-i \epsilon)^{2}}+\frac{1}{(2 k \lambda+i \epsilon)^{2}}\right] \int \frac{d^{D-1} p}{(2 \pi)^{D-1}} \frac{e^{i \mathbf{p} \cdot \mathbf{r}}}{p^{4}} \\
& \quad+\frac{e^{2}}{2} \int_{-1}^{1} d \lambda\left[\frac{1}{(2 k \lambda-i \epsilon)^{4}}+\frac{1}{(2 k \lambda+i \epsilon)^{4}}\right] \\
& \quad \times \int \frac{d^{D-1} p}{(2 \pi)^{D-1}} \frac{e^{i \mathbf{p} \cdot \mathbf{r}}}{p^{2}}-\frac{e^{2}}{2} \int_{-1}^{1} d \lambda \int \frac{d^{D-1} p}{(2 \pi)^{D-1}} \frac{e^{i \mathbf{p} \cdot \mathbf{r}}}{p} \\
& \quad \times\left[\frac{1}{(2 k \lambda-i \epsilon)^{4}(p-2 k \lambda+i \epsilon)}\right. \\
& \left.\quad+\frac{1}{(2 k \lambda+i \epsilon)^{4}(p-2 k \lambda-i \epsilon)}\right] .
\end{aligned}
$$


Some terms vanish because of the symmetry $\lambda \rightarrow-\lambda$. The integral over $p$ in the first and second terms is straightforward. By using the symmetries in the $\lambda$ and $p$ variables, the third term can be simplified as follows:

$$
\begin{aligned}
\frac{i e^{2}}{32 \pi^{2} r} \int_{-1}^{1} d \lambda \int_{-\infty}^{\infty} d p\left(e^{i p \cdot r}-e^{-i p \cdot r}\right) \\
\quad \times\left[\frac{1}{(2 k \lambda-i \epsilon)^{4}(p-2 k \lambda+i \epsilon)}\right. \\
+\frac{1}{(2 k \lambda+i \epsilon)^{4}(p+2 k \lambda+i \epsilon)} \\
+\frac{1}{(2 k \lambda+i \epsilon)^{4}(p-2 k \lambda-i \epsilon)} \\
\left.+\frac{1}{(2 k \lambda-i \epsilon)^{4}(p+2 k \lambda-i \epsilon)}\right] .
\end{aligned}
$$

At this point, the integral over $p$ can be done using standard techniques of complex analysis,

$$
\begin{aligned}
- & \frac{e^{2}}{8 \pi r} \int_{-1}^{1} d \lambda\left[\frac{e^{i 2 k \lambda}}{(2 k \lambda+i \epsilon)^{4}}+\frac{e^{-i 2 k \lambda}}{(2 k \lambda-i \epsilon)^{4}}\right] \\
= & -\frac{e^{2}}{8 \pi r} \int_{-1}^{1} d \lambda \cos (2 k \lambda)\left[\frac{1}{(2 k \lambda+i \epsilon)^{4}}+\frac{1}{(2 k \lambda-i \epsilon)^{4}}\right] \\
& -\frac{i e^{2}}{8 \pi r} \int_{-1}^{1} d \lambda \sin (2 k \lambda)\left[\frac{1}{(2 k \lambda+i \epsilon)^{4}}-\frac{1}{(2 k \lambda-i \epsilon)^{4}}\right] .
\end{aligned}
$$

Hence, our final result for Eq. (D12) reads

$$
\begin{aligned}
& -\frac{e^{2}}{8 \pi r} \int_{-1}^{1} d \lambda\left[\frac{1}{(2 k \lambda+i \epsilon)^{4}}+\frac{1}{(2 k \lambda-i \epsilon)^{4}}\right] \\
& \times\left[\cos (2 k \lambda r)-1+2 k^{2} \lambda^{2} r^{2}\right]-\frac{i e^{2}}{8 \pi r} \int_{-1}^{1} d \lambda \sin (2 k \lambda r) \\
& \quad \times\left[\frac{1}{(2 k \lambda+i \epsilon)^{4}}-\frac{1}{(2 k \lambda-i \epsilon)^{4}}\right]
\end{aligned}
$$

After performing the integration in $\lambda$, we obtain the second line and the first term of the third line in Eq. (22). $\Delta_{3}$ can be computed in a very similar way and leads to the second term in the first line of Eq. (22). Let us next consider $\Delta_{4}$,

$$
\begin{gathered}
\frac{4 i T e^{4}}{\pi} \int \frac{d^{D-1} p}{(2 \pi)^{D-1}} \frac{e^{i \mathbf{p} \cdot \mathbf{r}}}{p^{5}} \int_{p / 2}^{\infty} \frac{d k k}{e^{\beta \sqrt{k^{2}+m_{e}^{2}}}+1} \\
=\frac{4 i T e^{4}}{\pi} \int \frac{d^{D-1} p}{(2 \pi)^{D-1}} \frac{e^{i \mathbf{p} \cdot \mathbf{r}}}{p^{5}}\left\{\int_{0}^{\infty} \frac{d k k}{e^{\beta \sqrt{k^{2}+m_{e}^{2}}}+1}\right. \\
-\frac{p^{2}}{8\left(e^{\beta m_{e}}+1\right)}+\left[\int_{p / 2}^{\infty} \frac{d k k}{e^{\beta \sqrt{k^{2}+m_{e}^{2}}}+1}\right. \\
\left.\left.-\int_{0}^{\infty} \frac{d k k}{e^{\beta \sqrt{k^{2}+m_{e}^{2}}}+1}+\frac{p^{2}}{8\left(e^{\beta m_{e}}+1\right)}\right]\right\} .
\end{gathered}
$$

Earlier, we separated the pieces that led to IR divergences from the ones that did not. The first term inside the curly brackets gives the second term in the third line in Eq. (22), and the second term together with $V_{m}$ gives the fourth line of Eq. (22). The rest of the terms in the curly brackets give the fifth line and the beginning of sixth line of Eq. (22). $\Delta_{5}$ can be computed in a very similar way and gives the remaining terms of Eq. (22).

\section{APPENDIX E: COMPUTATION OF THE HTL RETARDED SELF-ENERGY FOR THE LONGITUDINAL PHOTON IN THE $m_{e} \neq 0$ CASE}

There are some subtle points in the computation that lead to Eq. (26), which do not arise in the massless case and are worth elaborating upon. Let us start from formula (37) of Ref. [31] for the massive case,

$$
\begin{aligned}
\Pi_{R}^{L}(P)= & -2 i e^{2} \int \frac{d^{D} k}{(2 \pi)^{D}}\left(q_{0} k_{0}+\mathbf{q} \cdot \mathbf{k}+m_{e}^{2}\right)\left[\tilde{\Delta}_{S}(Q) \tilde{\Delta}_{R}(K)\right. \\
& \left.+\tilde{\Delta}_{A}(Q) \tilde{\Delta}_{S}(K)\right],
\end{aligned}
$$

$Q=P-K$. It is customary to make the change $K \rightarrow-Q$ in the second term to get a simplified expression that reduces to twice the first term. However, the terms proportional to $m_{e}^{2}$, which do not exist in the massless case, have a stronger IR sensibility than the remaining ones. This leads to ill-defined expressions in the HTL approximation for $T \sim m_{e}$. These expressions must be properly defined in order to get consistent results before and after the shift $K \rightarrow-Q$ has been carried out.

To see this in detail, we work out just a small part of the computation, which illustrates the point, namely, the part of $\operatorname{Im} \Pi_{R}^{L}(P)$ that comes only from the $m_{e}^{2}$ term in the numerator. We call this term $\Pi_{m_{e}^{2}}(P)$,

$$
\begin{aligned}
\Pi_{m_{e}^{2}}(P)= & -2 i e^{2} m_{e}^{2} \int \frac{d^{D} k}{(2 \pi)^{D}}\left[\tilde{\Delta}_{S}(Q) \tilde{\Delta}_{R}(K)\right. \\
& \left.+\tilde{\Delta}_{A}(Q) \tilde{\Delta}_{S}(K)\right] .
\end{aligned}
$$

One can use the shift $K \rightarrow-Q$ to get a simplified expression,

$$
\Pi_{m_{e}^{2}}(P)=-4 i e^{2} m_{e}^{2} \int \frac{d^{D} k}{(2 \pi)^{D}} \tilde{\Delta}_{A}(Q) \tilde{\Delta}_{S}(K) .
$$

By carrying out the HTL expansion and by taking into account that we must also expand $p_{0}\left(p \gg p_{0}\right.$ in the computation of the potential), we obtain

$$
\begin{aligned}
\Pi_{m_{e}^{2}}(P)= & 8 e^{2} m_{e}^{2} p^{2} \int \frac{d^{D-1} k}{(2 \pi)^{D-1}} \frac{n_{F}\left(\sqrt{k^{2}+m_{e}^{2}}\right)}{\sqrt{k^{2}+m_{e}^{2}}} \\
& \times\left[\frac{1}{(2 \mathbf{k} \cdot \mathbf{p}-i \epsilon)^{2}}+\frac{4 p_{0} \sqrt{k^{2}+m_{e}^{2}}}{(2 \mathbf{k} \cdot \mathbf{p}-i \epsilon)^{3}}+\cdots\right] .
\end{aligned}
$$

Notice that the last term is ill defined in the IR (this is apparent if spherical coordinates are used). Let us focus on the imaginary part,

$$
\begin{aligned}
\operatorname{Im} \Pi_{m_{e}^{2}}= & -16 i e^{2} m_{e}^{2} p_{0} p^{2} \int \frac{d^{D-1} k}{(2 \pi)^{D-1}} n_{F}\left(\sqrt{k^{2}+m_{e}^{2}}\right) \\
& \times\left[\frac{1}{(2 \mathbf{k} \cdot \mathbf{p}-i \epsilon)^{3}}-\frac{1}{(2 \mathbf{k} \cdot \mathbf{p}+i \epsilon)^{3}}\right] .
\end{aligned}
$$

For illustration purposes, we will make this computation in two ways

(1) Using $d^{D-1} k=d \Omega_{D-2} d(\cos \theta) k^{D-2} d k$, where $\theta$ is the angle between the internal momentum and the external 
one,

$$
\begin{aligned}
\operatorname{Im} \Pi_{m_{e}^{2}}= & -32 i e^{2} m_{e}^{2} p_{0} p^{2} \frac{\Omega_{D-2}}{(2 \pi)^{D-1}} \\
& \times \int_{0}^{\infty} d k k^{D-2} n_{F}\left(\sqrt{k^{2}+m_{e}^{2}}\right) \\
& \times \int_{0}^{\pi} \frac{d(\cos \theta)}{(2 k p \cos \theta-i \epsilon)^{3}},
\end{aligned}
$$

and performing the angular integration, one arrives at

$$
\begin{aligned}
\operatorname{Im} \Pi_{m_{e}^{2}}= & 4 \pi e^{2} \frac{m_{e}^{2} p_{0}}{p} \frac{\Omega_{D-2}}{(2 \pi)^{D-1}} \\
& \times \int_{0}^{\infty} d k n_{F}\left(\sqrt{k^{2}+m_{e}^{2}}\right) k^{D-4} \delta(k) .
\end{aligned}
$$

This expression has an end-point singularity, but one can skip it in DR by choosing $D-4>0$. So, the result is zero in this way.

(2) Using $d^{D-1} k=d^{D-2} k_{\perp} d k_{z}$, we choose $z$ to be the direction parallel to the external momenta,

$$
\begin{aligned}
\operatorname{Im} \Pi_{m_{e}^{2}}= & -16 i e^{2} m_{e}^{2} p_{0} p^{2} \frac{\Omega_{D-2}}{(2 \pi)^{D-1}} \\
& \times \int_{0}^{\infty} d k_{\perp} k_{\perp}^{D-3} n_{F}\left(\sqrt{k_{\perp}^{2}+k_{z}^{2}+m_{e}^{2}}\right) \\
& \times \int_{-\infty}^{\infty} d k_{z}\left[\frac{1}{\left(2 p k_{z}-i \epsilon\right)^{3}}-\frac{1}{\left(2 p k_{z}+i \epsilon\right)^{3}}\right] .
\end{aligned}
$$

Notice that the integrand vanishes for all $k_{z}$ except when $k_{z} \sim$ 0 , so one may substitute

$$
n_{F}\left(\sqrt{k_{\perp}^{2}+k_{z}^{2}+m_{e}^{2}}\right) \rightarrow n_{F}\left(\sqrt{k_{\perp}^{2}+m_{e}^{2}}\right)+\frac{d n_{F}}{d E} \frac{k_{z}^{2}}{2 \sqrt{k_{\perp}^{2}+m_{e}^{2}}} .
$$

This simplifies the computation a lot because complex plane integration techniques can be applied,

$$
\begin{aligned}
\operatorname{Im} \Pi_{m_{e}^{2}}= & 4 \pi e^{2} m_{e}^{2} p_{0} \frac{\Omega_{D-2}}{(2 \pi)^{D-1}} \int_{0}^{\infty} \frac{d k_{\perp} k_{\perp}^{D-3}}{\sqrt{k_{\perp}^{2}+m_{e}^{2}}} \frac{d n_{F}}{d E} \\
& \times \int_{-\infty}^{\infty} d k_{z} \delta\left(2 p k_{z}\right) .
\end{aligned}
$$

This expression does not have any end-point singularity, and, in fact, it does not need a regularization anymore,

$$
\operatorname{Im} \Pi_{m_{e}^{2}}=-\frac{e^{2} m_{e}^{2} p_{0}}{2 \pi p\left(e^{\beta m_{e}}+1\right)} .
$$

Indeed, this is the expected result, which eventually leads to terms contributing to the second line of Eq. (26). Then, we arrive at the paradoxical situation in which the final result depends on the precise way DR is implemented, either in spherical coordinates or in Cartesian ones. The apparent contradiction is resolved by noticing that DR in spherical coordinates does not allow for the shift $K \rightarrow-Q$. In order to show this is the actual reason for it, let us start now from Eq. (E2),

$$
\begin{aligned}
\operatorname{Im} & \Pi_{m_{e}^{2}}(P) \\
= & -4 \pi^{2} e^{2} m_{e}^{2} \int \frac{d^{D} k}{(2 \pi)^{D}} \delta\left(K^{2}-m_{e}^{2}\right) \\
& \times \delta\left((K-P)^{2}-m_{e}^{2}\right)\left\{\operatorname{sgn}\left(k_{0}-p_{0}\right)\left[1-2 n_{F}\left(\left|k_{0}\right|\right)\right]\right. \\
& \left.-\operatorname{sgn}\left(k_{0}\right)\left[1-2 n_{F}\left(\left|k_{0}-p_{0}\right|\right)\right]\right\} .
\end{aligned}
$$

The integral over $k_{0}$ is straightforward,

$$
\begin{aligned}
\operatorname{Im} & \Pi_{m_{e}^{2}}(P) \\
= & -2 e^{2} m_{e}^{2} \pi \int \frac{d^{D-1} k}{(2 \pi)^{D-1}} \frac{1}{\sqrt{k^{2}+m_{e}^{2}}} \\
& \times\left\{\left[n_{F}\left(\sqrt{k^{2}+m_{e}^{2}}-p_{0}\right)-n_{F}\left(\sqrt{k^{2}+m_{e}^{2}}\right)\right]\right. \\
& \times \delta\left(p_{0}^{2}-p^{2}-2 p_{0} \sqrt{k^{2}+m_{e}^{2}}+2 \mathbf{k} \cdot \mathbf{p}\right) \\
& -\left[n_{F}\left(\sqrt{k^{2}+m_{e}^{2}}+p_{0}\right)-n_{F}\left(\sqrt{k^{2}+m_{e}^{2}}\right)\right] \\
& \left.\times \delta\left(p_{0}^{2}-p^{2}+2 p_{0} \sqrt{k^{2}+m_{e}^{2}}+2 \mathbf{k} \cdot \mathbf{p}\right)\right\} .
\end{aligned}
$$

So far, we have used the complete expression for the self-energy. Next, we apply HTL expansion and also $p \gg p_{0}$, as earlier,

$$
\begin{aligned}
\operatorname{Im} \Pi_{m_{e}^{2}}(P)= & -2 e^{2} m_{e}^{2} \pi \int \frac{d^{D-1} k}{(2 \pi)^{D-1}} \\
& \times \frac{2 p_{0} e^{\beta \sqrt{k^{2}+m_{e}^{2}}}}{T \sqrt{k^{2}+m_{e}^{2}}\left(e^{\beta \sqrt{k^{2}+m_{e}^{2}}}+1\right)^{2}} \delta(2 \mathbf{k} \cdot \mathbf{p}) .
\end{aligned}
$$

From this expression, no matter if one uses spherical or Cartesian coordinates, one arrives at

$$
\operatorname{Im} \Pi_{m_{e}^{2}}=-\frac{e^{2} m_{e}^{2} p_{0}}{2 \pi p\left(e^{\beta m_{e}}+1\right)},
$$

which agrees with Eq. (E11). If the expansion for small $p_{0}$ is not carried out, the same result is found with the three methods because then no regularization is needed.

The conclusion is that when the formula for the retarded self-energy in the massive case [1], that is,

$$
\begin{aligned}
\Pi_{R}^{L}(P)= & 4 e^{2} \int \frac{d^{D-1} k}{(2 \pi)^{3} \sqrt{k^{2}+m_{e}^{2}}} \frac{1}{e^{\beta \sqrt{k^{2}+m_{e}^{2}}}+1} \\
& \times \frac{p^{2}-\frac{(\mathbf{p} \cdot \mathbf{k})^{2}}{k^{2}+m_{e}^{2}}}{\left(p_{0}-\frac{\mathbf{p} \cdot \mathbf{k}}{\sqrt{k^{2}+m_{e}^{2}}}+i \epsilon\right)^{2}}
\end{aligned}
$$

is expanded for $p \gg p_{0}$, DR must be used in Cartesian coordinates in order to properly regulate IR divergences (i.e., to be consistent with the shift of momenta carried out at some point in order to get the preceding expression). This point was overlooked when calculating the potential in formula (57) of Ref. [1], and terms analogous to Eq. (E14) were missed. The correct formula is obtained by making the following substitution in Eq. (57) of Ref. [1]:

$$
g(m \beta) \rightarrow g(m \beta)+\frac{m^{2} \beta^{2}}{2\left(e^{\beta m}+1\right)} .
$$


[1] M. A. Escobedo and J. Soto, Phys. Rev. A 78, 032520 (2008).

[2] M. Laine, O. Philipsen, P. Romatschke, and M. Tassler, J. High Energy Phys. 03 (2007) 054.

[3] M. Laine, J. High Energy Phys. 05 (2007) 028.

[4] Y. Burnier, M. Laine, and M. Vepsäläinen, J. High Energy Phys. 01 (2008) 043.

[5] A. Beraudo, J. P. Blaizot, and C. Ratti, Nucl. Phys. A 806, 312 (2008).

[6] N. Brambilla, J. Ghiglieri, A. Vairo, and P. Petreczky, Phys. Rev. D 78, 014017 (2008).

[7] Y. Burnier, M. Laine, and M. Vepsäläinen, J. High Energy Phys. 02 (2009) 008.

[8] F. Kottmann et al., Hyperfine Interact. 138, 55 (2001).

[9] A. Di Giacomo, Nucl. Phys. B 11, 411 (1969).

[10] R. Pohl et al., Nature (London) 466, 213 (2010).

[11] V. G. Ivanov, E. Y. Korzinin, and S. G. Karshenboim, Phys. Rev. A 80, 022510 (2009).

[12] E. Borie, Phys. Rev. A 71, 032508 (2005).

[13] T. Kinoshita and M. Nio, Phys. Rev. D 60, 053008 (1999).

[14] T. Kinoshita and M. Nio, Phys. Rev. Lett. 82, 3240 (1999).

[15] K. Pachucki, Phys. Rev. A 53, 2092 (1996).

[16] A. Pineda, Phys. Rev. C 67, 025201 (2003).

[17] M. Bregant et al., Phys. Lett. A 241, 344 (1998).

[18] M. C. Fujiwara et al. (TRIUMF Muonic Hydrogen Collaboration), The Hydrogen Atom-Precision Physics with Simple Atomic Systems (Springer, Berlin, 2001).

[19] B. Shen and J. Meyer-ter-Vehn, Phys. Rev. E 65, 016405 (2001).

[20] A. G. Aksenov, R. Ruffini, and G. V. Vereshchagin, Phys. Rev. D 79, 043008 (2009).
[21] A. G. Aksenov, R. Ruffini, and G. V. Vereshchagin, Phys. Rev. Lett. 99, 125003 (2007).

[22] M. H. Thoma, Rev. Mod. Phys. 81, 959 (2009).

[23] L. Hollberg and J. L. Hall, Phys. Rev. Lett. 53, 230 (1984).

[24] J. P. Blaizot and E. Iancu, Phys. Rep. 359, 355 (2002).

[25] D. H. Rischke, Prog. Part. Nucl. Phys. 52, 197 (2004).

[26] M. H. Thoma, J. Phys. A 42, 214004 (2009).

[27] W. E. Caswell and G. P. Lepage, Phys. Lett. B 167, 437 (1986).

[28] A. Pineda and J. Soto, Nucl. Phys. Proc. Suppl. 64, 428 (1998).

[29] E. Braaten and R. D. Pisarski, Phys. Rev. D 46, 1829 (1992).

[30] M. Le Bellac, Thermal Field Theory (Cambridge University Press, Cambridge, UK, 1996), 256 p.

[31] M. H. Thoma, e-print arXiv:hep-ph/0010164.

[32] A. Pineda, Phys. Rev. A 66, 062108 (2002).

[33] A. Pineda and J. Soto, Phys. Lett. B 420, 391 (1998).

[34] A. V. Manohar, Phys. Rev. D 56, 230 (1997).

[35] U. D. Jentschura and M. Haas, Phys. Rev. A 78, 042504 (2008).

[36] M. Beneke and V. A. Smirnov, Nucl. Phys. B 522, 321 (1998).

[37] V. A. Smirnov, Springer Tracts Mod. Phys. 177, 1 (2002).

[38] N. Brambilla, M. A. Escobedo, J. Ghiglieri, J. Soto, and A. Vairo, J. High Energy Phys. 09 (2010) 038.

[39] D. Bodeker, e-print arXiv:hep-ph/9909375.

[40] M. Laine, O. Philipsen, and M. Tassler, J. High Energy Phys. 09 (2007) 066.

[41] D. Eiras and J. Soto, Phys. Lett. B 491, 101 (2000).

[42] A. Pineda, Ph.D. thesis, Universitat de Barcelona, 1998. 